J. DIFFERENTIAL GEOMETRY

50 (1998) $25-58$

\title{
A SCHLÄFLI-TYPE FORMULA FOR CONVEX CORES OF HYPERBOLIC 3-MANIFOLDS
}

\author{
FRANCIS BONAHON
}

Dedicated to D.B.A. Epstein, on his 60th birthday.

Let $M$ be a (connected) hyperbolic 3-manifold, namely a complete Riemannian manifold of dimension 3 and of constant sectional curvature -1 , with finitely generated fundamental group. We exclude the somewhat degenerate case where $\pi_{1}(M)$ has an abelian subgroup of finite index. A fundamental subset of $M$ is its convex core $C_{M}$, which is the smallest non-empty convex subset of $M$. The condition that the volume of $C_{M}$ is finite is open in the space of hyperbolic metrics on $M$, provided we restrict attention to cusp-respecting deformations. In this paper, we give a formula which, for a cusp-preserving variation of the hyperbolic metric of $M$, expresses the variation of the volume of the convex core $C_{M}$ in terms of the variation of the bending measure of its boundary.

This formula is analogous to the Schläfli formula for the volume of an $n$-dimensional hyperbolic polyhedron $P$; see [19], [12], [1] and $\S 1$. If the metric of $P$ varies, the Schläfli formula expresses the variation of the volume of $P$ in terms of the variation of the dihedral angles of $P$ along the $(n-2)$-faces of its boundary and of the $(n-2)$-volumes of these faces.

Received May 16, 1997. This research was partially supported by N.S.F. grants DMS-9001895, DMS-9201466 and DMS-9504282.

Keywords and phrases. hyperbolic geometry, convex hull, convex core, hyperbolic volume

1991 Mathematics Subject Classification. 53C25, 30F40, 57N05 
The analogy stems from the fact that the boundary $\partial C_{M}$ of $C_{M}$ is almost polyhedral, in the sense that it is totally geodesic almost everywhere. However, the pleating locus, where $\partial C_{M}$ is not totally geodesic, is not a finite collection of edges any more. Typically, it will consist of uncountably many infinite geodesics. In addition, the topology of this pleating locus can drastically change as we vary the metric of $M$. So the situation is much more complex.

The path metric induced on the surface $\partial C_{M}$ by the metric of $M$ is hyperbolic with finite area. On this hyperbolic surface, the pleating locus $\lambda$ forms a compact geodesic lamination, namely is compact and is the union of disjoint simple geodesics. The surface $\partial C_{M}$ is bent along $\lambda$, and the amount of this bending can be measured, not by dihedral angles any more, but by a transverse measure for $\lambda$. Endowing $\lambda$ with this transverse measure, we get a measured lamination $b$, called the bending measured lamination of $M$; see [21], [10].

Let $M$ be a hyperbolic $3-$ manifold which is geometrically finite, namely such that the convex core $C_{M}$ has finite volume and the fundamental group $\pi_{1}(M)$ is finitely generated. Consider a deformation of $M$, namely a differentiable 1 -parameter family of hyperbolic manifolds $M_{t}, t \in\left[0, \varepsilon\left[\right.\right.$, such that $M_{0}=M$; when $M$ has cusps, we also require that the cusps of each $M_{t}$ precisely correspond to the cusps of $M$. Then, $M_{t}$ is also geometrically finite for $t$ small enough [13]. We showed in [6] that, if $b_{t}$ is the bending measured lamination of $M_{t}$, then the family $b_{t}, t \in\left[0, \varepsilon\left[\right.\right.$, admits a tangent vector $\dot{b}_{0}$ at $t=0$, in the piecewise linear manifold $\mathcal{M L}\left(\partial C_{M}\right)$ of all measured geodesic laminations on $\partial C_{M}$. In addition, in [3], [4], we showed that such a tangent vector can be geometrically interpreted as a geodesic lamination endowed with a certain type of transverse distribution, called a transverse Hölder distribution.

On a hyperbolic surface, a geodesic lamination with transverse distribution $a$ admits a certain length [4], [5]. This length is designed so that it varies continuously with $a$ and coincides with the usual length when $a$ consists of a simple closed geodesic endowed with the Dirac transverse distribution. In particular, on the hyperbolic surface $\partial C_{M_{0}}$, we can consider the length $l_{0}\left(\dot{b}_{0}\right)$ of the tangent vector $\dot{b}_{0}$.

Main Theorem. With the above data, the volume $V_{t}$ of the convex core $C_{M_{t}}$ admits a right derivative $\dot{V}_{0}$ at $t=0$, and

$$
\dot{V}_{0}=\frac{1}{2} l_{0}\left(\dot{b}_{0}\right)
$$

where $l_{0}\left(\dot{b}_{0}\right)$ is the length of the vector $\dot{b}_{0}$ tangent to the family of bending 
measured laminations $b_{t}$.

In the case of a differentiable deformation $\left.M_{t}, t \in\right]-\varepsilon, \varepsilon[$, the right and left derivatives of the volume of $C_{M_{t}}$ may not necessarily agree at $t=0$, as shown for instance by the example of $[6, \S 6]$.

An application of this theorem is the following corollary, proved in $\S 4$.

Corollary. Given a geometrically finite hyperbolic 3-manifold $M$, consider the volumes of the convex cores of the cusp-preserving deformations of $M$. If the boundary of the convex core $C_{M}$ is totally geodesic, then $M$ corresponds to a local minimum of this volume function.

We prove the Main Theorem in two steps. One step, proved in $\S 3$, is to show that the volume of the convex core of $M_{t}$ has the same right derivative at $t=0$ as the volume enclosed in $M_{t}$ by a pleated surface whose pleating locus is constant and contains the pleating locus of $\partial C_{M_{0}}$. This step heavily relies on the arguments of [6]. The other step, proved in $\S 2$, is devoted to a Schläfli formula for the volume enclosed by a pleated surface whose pleating locus is constant. This simpler formula is proved by cutting the enclosed volume into small pieces and applying the usual Schläfli formula to the pieces. The formal aspects of this part of the proof are relatively natural. However, much care is needed to justify these formal arguments, owing to the subtleties of the convergence of transverse distributions and to the fact that one has to estimate derivatives of dihedral angles, and not just dihedral angles. The cancellation of the contributions of internal edges is also non-trivial.

This article was written while the author was holding visiting positions at the Centre Émile Borel, the Institut des Hautes Études Scientifiques and the California Institute of Technology. He would like to thank these institutions for their productive hospitality. He is also grateful to Steve Carlip for asking the question which originally motivated his interest in these problems, and to Alberto Candel for help in the proof of Lemma 9 .

\section{The Schläfli formula for hyperbolic cycles in 3-manifolds}

The classical Schläfli Formula is a crucial tool in the proof of the Main Theorem. Although it holds in any dimension and in any space 
of non-zero constant curvature, we state it here only for hyperbolic 3dimensional geometry since this is the only case which we will use. See [19], [12], [1, Chap. 7, §2.2] for a proof.

Consider a differentiable 1-parameter family of hyperbolic polyhedra $P_{t}, t \in\left[0, \varepsilon\left[\right.\right.$, in hyperbolic 3 -space $\mathbb{H}^{3}$. This means that the polyhedra $P_{t}$ all have the same combinatorial type, that their faces and edges are totally geodesic in $\mathbb{H}^{3}$, and that their vertices vary differentiably with $t$.

Theorem 1 (Schläfli Formula). Let $P_{t}, t \in[0, \varepsilon[$, be a differentiable 1 -parameter family of polyhedra in $\mathbb{H}^{3}$. Then the right derivative of the volume $V_{t}$ of $P_{t}$ at $t=0$ is

$$
\dot{V}_{0}=\frac{1}{2} \sum_{e \text { edge of } P_{0}} l_{0}(e) \dot{b}_{0}(e)
$$

where $l_{0}(e)$ denotes the length of the edge e in $P_{0}$, and $\dot{b}_{0}(e)$ is the right derivative at $t=0$ of the external dihedral angle $b_{t}(e)$ of $P_{t}$ along this same edge. q.e.d.

Here, the external dihedral angle $b_{t}(e)$ is $\pi$ minus the internal dihedral angle of $P_{t}$ at $e$. In particular, the external dihedral angle is equal to 0 when the boundary of $P_{t}$ is flat at $e$, and is equal to $\pi$ when the two faces that are adjacent to $e$ locally coincide near $e$.

There is a convenient notation, which already appeared in the Introduction and in the above statement, which we will consistently use throughout the paper: When a quantity $A_{t}$ depends on $t$, we will denote by $\dot{A}_{t_{0}}$ its right derivative with respect to $t$ at $t=t_{0}$. For instance, $\dot{b}_{0}(e)$ is the right derivative of $b_{t}(e)$ at $t=0$.

Also, before going any further, we should observe that it suffices to prove the Main Theorem for orientable manifolds. Indeed, passing to the orientation cover multiplies each side of the equality by 2 . Consequently, we will henceforth assume that all manifolds considered are orientable, and often oriented.

We can give a homological flavor to the formula of Theorem 1 in the following way. Let $M_{t}, t \in[0, \varepsilon[$, be a differentiable 1-parameter family of connected oriented hyperbolic 3-manifolds. By definition, the differentiability condition means that there is a fixed group $\Gamma$ and orientationpreserving discrete faithful representations $\rho_{t}: \Gamma \rightarrow \operatorname{Isom}^{+}\left(\mathbb{H}^{3}\right)$ into the isometry group of $\mathbb{H}^{3}$ such that each $M_{t}$ is isometric to $\mathbb{H}^{3} / \rho_{t}(\Gamma)$ and $\rho_{t}(\gamma)$ depends differentiably on $t$ for every $\gamma \in \Gamma$. Fix a compact triangulated surface $S$ without boundary, not necessarily connected, and 
consider polyhedral maps $f_{t}: S \rightarrow M_{t}$, namely continuous maps whose restriction to each edge and face of the triangulation of $S$ is a totally geodesic immersion. In addition, we require these maps $f_{t}: S \rightarrow M_{t}=$ $\mathbb{H}^{3} / \rho_{t}(\Gamma)$ to depend differentiably on $t$ in the sense that, if we lift them to maps $\widetilde{f}_{t}: \widetilde{S} \rightarrow \mathbb{H}^{3}$ defined on the universal covering $\widetilde{S}$ of $S$, the images of the vertices of $\widetilde{S}$ under $\widetilde{f_{t}}$ depend differentiably on $t$.

Corollary 2. Given a triangulated compact oriented surface $S$, let $f_{t}: S \rightarrow M_{t}, t \in[0, \varepsilon[$, be a differentiable 1-parameter family of polyhedral maps from $S$ to oriented hyperbolic 3-manifolds $M_{t}$. Assume that the $f_{t}$ are homologous to 0 in $M_{t}$ and that the $M_{t}$ are non-compact, so that the volume $V_{t}$ of a 3 -chain bounding $f_{t}$ in $M_{t}$ is well defined. Then the right derivative of the volume $V_{t}$ at $t=0$ is

$$
\dot{V}_{0}=\frac{1}{2} \sum_{e \text { edge of } S} l_{0}(e) \dot{b}_{0}(e)
$$

where, for each edge e of $S, l_{0}(e)$ denotes the length of $f_{0}(e)$ and $b_{t}(e)$ is the external angle between the two faces of $f_{t}(S)$ meeting along $f_{t}(e)$.

Proof. Since $f_{0}$ is homologous to 0 , we can extend it to a map $f_{0}: \Sigma \rightarrow M_{0}$ where $\Sigma$ is a simplicial complex with boundary $S$. We can choose this extension to be polyhedral. Then, since the hyperbolic manifolds $M_{t}$ and the maps $f_{t}: S \rightarrow M_{t}$ depend differentiably on $t$, we can easily extend them to a differentiable 1-parameter family of polyhedral maps $f_{t}: \Sigma \rightarrow M_{t}$ for $t$ small enough.

Let $P_{1}, \ldots, P_{n}$ be the 3 -simplices of $\Sigma$. Apply Theorem 1 to each hyperbolic 3-simplex $f_{t \mid P_{i}}: P_{i} \rightarrow \mathbb{H}^{3}$. Note that the volume $V_{t}^{i}$ of this simplex is negative when $f_{t \mid P_{i}}$ is orientation reversing. Then,

$$
\begin{aligned}
\dot{V}_{0} & =\sum_{i=1}^{n} \dot{V}_{0}^{i}=\frac{1}{2} \sum_{i=1}^{n} \sum_{e \text { edge of } P_{i}} l_{0}(e) \dot{b}_{0}^{i}(e) \\
& =\frac{1}{2} \sum_{e \text { edge of } \Sigma} l_{0}(e) \sum_{P_{i} \text { containing } e} \dot{b}_{0}^{i}(e)
\end{aligned}
$$

where $b_{t}^{i}(e)$ is the external angle between the two faces of $f_{t \mid P_{i}}$ meeting along the edge $f_{t \mid e}$ (counted negative if $f_{t \mid P_{i}}$ is orientation reversing).

For every edge $e$ of $\Sigma$ that is not in $S$,

$$
\sum_{P_{i} \text { containing } e} b_{t}^{i}(e) \equiv 0 \bmod \pi,
$$


and it follows that the corresponding derivative is equal to 0 . On the other hand, for every edge $e$ of $S$,

$$
\sum_{P_{i} \text { containing } e} b_{t}^{i}(e) \equiv b_{t}(e) \quad \bmod \pi .
$$

The formula of Corollary 2 immediately follows. q.e.d.

\section{Cycles bounded by pleated surfaces in hyperbolic 3-manifolds}

Let $M_{t}, t \in[0, \varepsilon[$, be a differentiable 1-parameter family of hyperbolic 3-manifolds, associated to the representations $\rho_{t}: \Gamma \rightarrow \operatorname{Isom}^{+}\left(\mathbb{H}^{3}\right)$. We require that this deformation of $M_{0}$ is cusp-preserving, in the sense that every element of $\Gamma$ which is sent to a parabolic element by $\rho_{0}$ is also sent to a parabolic element by each $\rho_{t}$. We also assume that $M_{0}$ is geometrically finite, namely that $\Gamma$ is finitely generated and that the convex core $C_{M_{0}}$ has finite volume. Then, the same also holds for every $C_{M_{t}}$ with $t$ small enough $[13, \S 9]$. In addition, the topology of $M_{t}$ and $\partial C_{M_{t}}$ remains constant for $t$ small enough, provided we use the following convention: When $C_{M_{0}}$ is 2-dimensional, namely when the group $\rho_{0}(\Gamma) \subset \operatorname{Isom}^{+}\left(\mathbb{H}^{3}\right)$ respects a hyperbolic plane in $\mathbb{H}^{3}$, we define $\partial C_{M_{0}}$ as the orientation covering of $C_{M_{0}}$, namely as the two sides of $C_{M_{0}}$ in $M_{0}$ (in contrast to the topological convention for which $\partial C_{M_{0}}$ should be equal to $C_{M_{0}}$ in this case).

We want to compute the variation of the volume of the convex core $C_{M_{t}}$, namely of the part of $M_{t}$ bounded by the pleated surface $\partial C_{M_{t}}$. As $t$ varies, the pleating locus of $\partial C_{M_{t}}$ usually changes, which is a source of technical difficulties. In this section, as a first step towards our goal, we consider a simpler situation by substituting to $\partial C_{M_{t}}$ a pleated surface in $M_{t}$ whose pleating locus is independent of $t$, and by considering the variation of the volume bounded by this pleated surface. The fact that the pleating locus is constant makes the situation reminiscent of that of the classical Schläfli formula of Theorem 1.

Let $S$ be an oriented surface of finite topological type, without boundary but possibly infinite and not necessarily connected. Consider a pleated surface $f_{0}: S \rightarrow M_{0}$. We refer to [21], [8, §5] for basic facts about pleated surfaces. In particular, an important convention is that $f_{0}$ is proper, and sends each end of $S$ to a cusp of $M_{0}$; as a consequence, the hyperbolic metric $m_{0}$ of $S$ obtained from the metric of $M_{0}$ by pull 
back under $f_{0}$ has finite volume, and each end of $S$ corresponds to a cusp of this metric. Although this is not absolutely necessary (see Remark 2 at the end of this section), we also require that $f_{0}$ is totally geodesic near the ends of $S$.

Let $\lambda$ be a pleating locus for $f_{0}$, namely a compact geodesic lamination in $S$ such that $f_{0}$ sends each leaf of $\lambda$ to a geodesic of $M_{0}$ and such that $f_{0}$ is a totally geodesic immersion on $S-\lambda$. Such a pleating locus may not be unique; an extreme example occurs when $f_{0}$ is totally geodesic, in which case every compact geodesic lamination in $S$ is a pleating locus for $f_{0}$. Increasing $\lambda$ without loss of generality (compare $[8, \S 4]$ ), we can assume that $\lambda$ is maximal among compact geodesic laminations, namely that every component of $S-\lambda$ is either an ideal triangle, bounded by 3 leaves of $\lambda$, or an open annulus bounded on one side by a leaf of $\lambda$ with one spike and leading to a cusp on the other side. Then, for every $t$ small enough, there is a unique pleated surface $f_{t}: S \rightarrow M_{t}$ with pleating locus $\lambda$ such that, for every leaf $g$ of $\lambda, f_{t}(g)$ is the geodesic of $M_{t}$ that is asymptotic to the image of $f_{0}(g)$ under the quasi-isometric homeomorphism $\varphi_{t}: M_{0} \rightarrow M_{t}$; see [21], [8,§5.3].

In [5], we describe the local geometry of the pleated surface $f_{t}$ by the pull back hyperbolic metric it induces on $S$, as well as a bending transverse cocycle $b_{t} \in \mathcal{H}(\lambda ; \mathbb{R} / 2 \pi \mathbb{Z})$ for the geodesic lamination $\lambda$, valued in $\mathbb{R} / 2 \pi \mathbb{Z}$, which measures the bending of the pleated surface $f_{t}$. This bending transverse cocycle associates a number $b_{t}(k) \in \mathbb{R} / 2 \pi \mathbb{Z}$ to each arc $k$ transverse to $\lambda$, which measures the bending of the pleated surface $f_{t}$ along the leaves of $\lambda$ meeting $k$. This $b_{t}(k)$ is invariant under homotopy of $k$ respecting $\lambda$, and behaves additively if we split $k$ into two subarcs. We also prove in [5] that this bending cocycle depends differentiably of the representation $\rho_{t}: \Gamma \rightarrow \operatorname{Isom}^{+}\left(\mathbb{H}^{3}\right)$ associated to $M_{t}$. In particular, there exists a right derivative $\dot{b}_{0} \in \mathcal{H}(\lambda ; \mathbb{R})$, which is an $\mathbb{R}$-valued transverse cocycle for $\lambda$.

In [3], we showed that every real-valued transverse cocycle $b \in$ $\mathcal{H}(\lambda ; \mathbb{R})$ defines a transverse distribution for $\lambda$. In particular, given a finite area hyperbolic metric $m$ on $S$, we can define a length $l_{m}(b) \in \mathbb{R}$ by, first making $\lambda$ an $m$-geodesic lamination, and then locally integrating with respect to the transverse distribution associated to $b$ the 1-dimensional Lebesgue measure along the leaves of $\lambda$; see [4], [5].

Finally, we assume that the pleated surfaces $f_{t}$ separate $M_{t}$. When $S$ is non-compact, this means that the locally finite 2 -chain defined by $f_{t}$ bounds a locally finite 3 -chain. We also require that this 3 -chain 
has finite volume $V_{t}$. If $M_{t}$ has infinite volume, this finite volume $V_{t}$ is uniquely defined, namely is independent to the finite volume 3 -chain bounding $f_{t}$. If $M_{t}$ has finite volume, $V_{t}$ is defined only modulo the volume of $M_{t}$; however Mostow's Rigidity Theorem implies that the $M_{t}$ and $f_{t}$ are independent of $t$ up to isometry, so that the theorem below is trivial in this case.

Theorem 3. Given an oriented surface $S$ of finite topological type, let $f_{t}: S \rightarrow M_{t}, t \in[0, \varepsilon[$, be a differentiable 1-parameter family of pleated surfaces in oriented geometrically finite hyperbolic 3-manifolds $M_{t}$, with pleating locus a fixed compact geodesic lamination $\lambda$ in $S$. Assume that $f_{t}$ bounds a finite volume (locally finite) 3-chain in $M_{t}$ and that $M_{t}$ has infinite volume, so that we can consider the volume $V_{t}$ of an arbitrary chain bounded by $f_{t}$ in $M_{t}$. Then,

$$
\dot{V}_{0}=\frac{1}{2} l_{0}\left(\dot{b}_{0}\right)
$$

where $b_{t} \in \mathcal{H}(\lambda ; \mathbb{R} / 2 \pi \mathbb{Z})$ is the bending cocycle of the pleated surface $f_{t}$, and the right-hand term denotes one half of the length of $\dot{b}_{0} \in \mathcal{H}(\lambda ; \mathbb{R})$ with respect to the hyperbolic metric $m_{0}$ on $S$ defined by pull back under $f_{0}$ of the hyperbolic metric of $M_{0}$.

Proof of Theorem 3 when there are no cusps. As traditional in 3dimensional hyperbolic geometry, the presence of cusps introduces some local technicalities which are not difficult, but tend to dilute attention away from the main points of the proof. For this reason, we will first restrict ourselves to the case where the hyperbolic manifolds $M_{t}$ have no cusps, and we will later explain how to extend the proof in the presence of cusps.

Consequently, assume that the surface $S$ is compact and that the manifolds $M_{t}$ have no cusps.

For every $t$, let $m_{t}$ be the hyperbolic metric on $S$ obtained by pulling back the metric of $M_{t}$ under $f_{t}$, and let $\lambda_{t}$ denote the $m_{t}$-geodesic lamination of $S$ corresponding to the geodesic lamination $\lambda$. By hypothesis, $\lambda$ is a maximal geodesic lamination, and the complement $S-\lambda_{t}$ consists of ideal triangles.

We can cover $\lambda_{0}$ by a family of rectangles $R_{1}^{(0)}, R_{2}^{(0)}, \ldots, R_{m}^{(0)}$ with $m_{0}$-geodesic sides, with disjoint interiors, and such that the components of $\lambda_{0} \cap R_{i}^{(0)}$ are all parallel to (and disjoint from) two opposite sides of the rectangle $R_{i}^{(0)}$, for each $i$. These rectangles more or less form a train track carrying $\lambda_{0}$. If we collapse each rectangle $R_{i}^{(0)}$ to an edge that is 
parallel to the components of $\lambda_{0} \cap R_{i}^{(0)}$, we obtain a graph embedded in $S$. Extend this graph to a triangulation $\mathcal{T}$ of $S$ and choose a map $g_{0}: S \rightarrow M_{0}$ which is homotopic to $f_{0}$, is polyhedral with respect to $\mathcal{T}$, and sends to a geodesic arc the image of each rectangle $R_{j}^{(0)}$ in the 1-skeleton of $\mathcal{T}$.

For $t$ small, we similarly construct rectangles $R_{1}^{(t)}, R_{2}^{(t)}, \ldots, R_{m}^{(t)}$ with $m_{t}$-geodesic sides and disjoint interiors such that the components of each $\lambda_{t} \cap R_{i}^{(t)}$ are parallel to one side of $R_{i}^{(t)}$. In addition, we require these $R_{i}^{(t)}$ to vary differentiably with $t$. In particular, collapsing the $R_{i}^{(t)}$ defines the same graph embedded in $S$, up to isotopy. Choose a map $g_{t}: S \rightarrow M_{t}$ that is homotopic to $f_{t}$, is polyhedral with respect to the triangulation $\mathcal{T}$, sends the image of each rectangle $R_{j}^{(t)}$ under the collapsing process to a geodesic arc, and varies differentiably with $t$.

The Schläfli formula of Corollary 2 determines the variation of the volume enclosed by the polyhedral map $g_{t}$. To compute the variation of the volume enclosed by $f_{t}$, it is therefore sufficient to analyze the volume of a homotopy between $f_{t}$ and $g_{t}$. In the definition of the triangulation $\mathcal{T}$, we implicitly used a map $h_{t}: S \rightarrow S$ homotopic to the identity and collapsing each rectangle $R_{i}^{(t)}$ to an arc contained in the 1-skeleton of $\mathcal{T}$. Since there is a volume 0 homotopy between $g_{t}$ and $g_{t} \circ h_{t}$, it suffices to determine the volume of a homotopy $H_{t}: S \times[0,1] \rightarrow M_{t}$ such that $H_{t \mid S \times\{1\}}=f_{t}$ and $H_{t \mid S \times\{0\}}=g_{t} \circ h_{t}$.

Let us focus attention on a rectangle $R_{i}^{(t)}$. We 'straighten' the homotopy $H_{t}$ on $R_{i}^{(t)} \times[0,1]$ in the following way. Identify $R_{i}^{(t)}$ to a standard rectangle $[a, b] \times[c, d]$ by an orientation-preserving homeomorphism such that each component of $\lambda_{t} \cap R_{i}^{(t)}$ corresponds to an $\operatorname{arc}\{x\} \times[c, d]$. Cut each rectangle $\{x\} \times[c, d] \times[0,1]$ in $R_{i}^{(t)} \times[0,1]$ into two triangles along the diagonal line joining $(x, c, 1)$ to $(x, d, 0)$, foliate the upper triangle by line segments originating from $(x, d, 0)$, and foliate the lower rectangle by line segments originating from $(x, c, 0)$. This decomposes $R_{i}^{(t)} \times[0,1] \cong[a, b] \times[c, d] \times[0,1]$ into a family of arcs; see Figure 1. We can now deform the restriction of $H_{t}$ to $R_{i}^{(t)} \times[0,1]$ so that it sends each of these arcs to a geodesic arc.

By construction, $H_{t}\left(R_{i}^{(t)} \times\{1\}\right)$ is equal to $f_{t}\left(R_{i}^{(t)}\right)$, and

$$
H_{t}\left(R_{i}^{(t)} \times\{0\}\right)=g_{t} \circ h_{t}\left(R_{i}^{(t)}\right)
$$




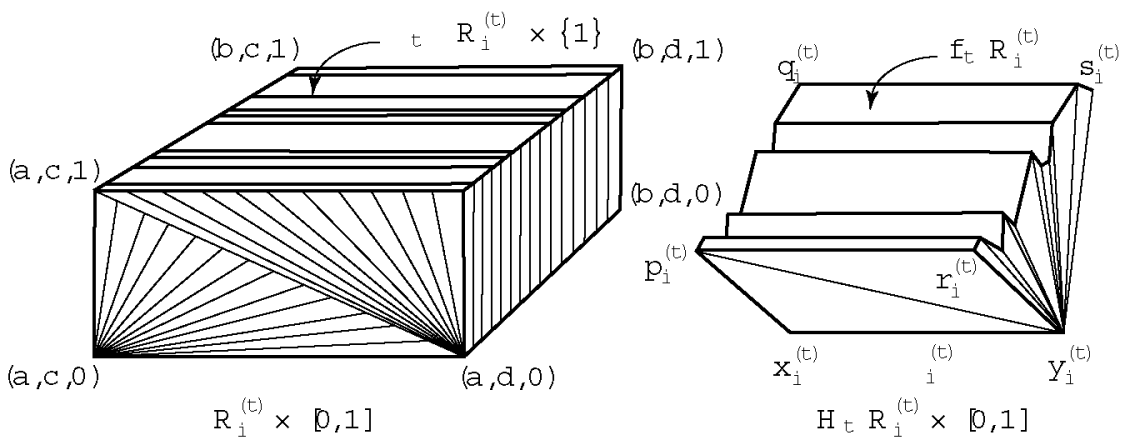

FIGURE 1

is a geodesic arc with end points

$$
x_{i}^{(t)}=H_{t}([a, b] \times\{c\} \times\{0\})
$$

and

$$
y_{i}^{(t)}=H_{t}([a, b] \times\{d\} \times\{0\}) .
$$

The face

$$
[a, b] \times\{c\} \times[0,1]
$$

of $R_{i}^{(t)} \times[0,1]$ is sent by $H_{t}$ to a 'pleated fan' which is the joint of the arc $f_{t}([a, b] \times\{c\})$ and the point $x_{i}^{(t)}$, namely $H_{t}([a, b] \times\{c\} \times[0,1])$ consists of all geodesic arcs that join $f_{t}([a, b] \times\{c\})$ to $x_{i}^{(t)}$ and are in the appropriate homotopy class. Similarly, $[a, b] \times\{d\} \times[0,1]$ is sent to the joint of $f_{t}([a, b] \times\{d\})$ and $y_{i}^{(t)}$. The remaining two faces $\{a\} \times[c, d] \times[0,1]$ and $\{b\} \times[c, d] \times[0,1]$ of $R_{i}^{(t)} \times[0,1]$ are each sent to the union of two totally geodesic triangles.

In particular, this analysis of the restriction of $H_{t}$ to the faces $[a, b] \times$ $\{c\} \times[0,1]$ and $[a, b] \times\{d\} \times[0,1]$ shows that we can globally deform $H_{t}$ so that it is of the above type on each rectangle $R_{i}^{(t)}$.

To evaluate the volume of the restriction of $H_{t}$ to $R_{i}^{(t)} \times[0,1]$, we decompose it into pieces. For each component $R$ of $R_{i}^{(t)}-\lambda_{t}$, $H_{t}(R \times[0,1])$ is the union of a pyramid with square basis, namely the joint of $f_{t}(R)$ and $y_{i}^{(t)}$, and of the tetrahedron formed by the joint of the two geodesic arcs $f_{t}(R \cap[a, b] \times\{c\})$ and $\gamma_{i}^{(t)}$. For each component 
$k$ of $\lambda_{t} \cap R_{i}^{(t)}, H_{t}(k \times[0,1])$ is the union of the two totally geodesic triangles which are, respectively, the joint of $f_{t}(k)$ and $y_{i}^{(t)}$ and the joint of $f_{t}(k \cap([a, b] \times\{c\}))$ and $\gamma_{i}^{(t)}$. For the metric $m_{t}, \lambda_{t}$ has 2-dimensional Lebesgue measure 0 in $S$, and $\lambda_{t} \cap[a, b] \times\{c\}$ has 1 -dimensional Lebesgue measure 0 in the transverse arc $[a, b] \times\{c\}$; see [9], [16], [2]. It follows that $H_{t}\left(\left(\lambda_{t} \cap R_{i}^{(t)}\right) \times[0,1]\right)$ has 3 -dimensional Lebesgue measure 0 . Therefore, we can focus only on the contribution of the components of $R_{i}^{(t)}-\lambda_{t}$

We can now sketch the proof of Theorem 3 . Let $R^{(t)} \subset S$ denote the union of the rectangles $R_{i}^{(t)}$. By construction, $H_{t}\left(\left(S-R^{(t)}\right) \times[0,1]\right)$ is bounded by a polyhedral surface, and the variation of its volume is given by Corollary 2 . We observed that the volume of $H_{t}\left(R^{(t)} \times[0,1]\right)$ is equal to the sum of the volumes of certain pyramids and tetrahedra. We can therefore expect that the variation of the volume of $H_{t}\left(R^{(t)} \times[0,1]\right)$ is equal to the sum of the variations of the volumes of these pyramids and tetrahedra, as given by Theorem 1. Altogether, this expresses the variation of the volume of $H_{t}$ as the sum of lengths of edges multiplied by the variation of dihedral angles at these edges. As in the proof of Corollary 2, the contributions of the internal edges cancel out, as well as the contribution of the edges that are contained in the sides of the rectangles $R_{i}^{(t)}$. This leaves the contributions of the edges of the polyhedral map $g_{t}$, which will cancel out with the variation of the volume enclosed by $g_{t}$, and the contribution of the edges contained in $f_{t}\left(\lambda_{t}\right)$, which can be re-interpreted as the length of the variation of the bending cocycle of the pleated surface $f_{t}$.

These ideas easily lead to a formal proof of Theorem 3 , but numerous points need to be justified. First of all, to be able to apply Corollary 2, we need to know that the shapes of the pyramids and tetrahedra of the decomposition are non-degenerate and vary differentiably with $t$. Then, because there are (in general) infinitely many such pyramids and tetrahedra, we have to show that the infinite sums involved do converge. Because the internal edges are not locally finite, the proof that their contributions cancel out is not as simple as in the proof of Corollary 2 . Finally, we have to identify the contribution of the edges contained in $f_{t}\left(\lambda_{t}\right)$ with the length of the variation of the bending cocycle of the pleated surface $f_{t}$.

Lemma 4. Given a component $R_{0}$ of $R_{i}^{(0)}-\lambda_{0}$, let $R_{t}$ be the corresponding component of $R_{i}^{(t)}-\lambda_{t}$. Then, the vertices of the rectangle 
$f_{t}\left(R_{t}\right)$ vary differentiably with $t$ in $M_{t}$.

Proof of Lemma 4. Recall that we are given a family of representations $\rho_{t}: \Gamma \rightarrow$ Isom $^{+}\left(\mathbb{H}^{3}\right)$ depending differentiably on $t$ and of isometries $M_{t} \cong \mathbb{H}^{3} / \rho_{t}(\Gamma)$. We want to show that $f_{t}\left(R_{t}\right) \subset M_{t} \cong \mathbb{H}^{3} / \rho_{t}(\Gamma)$ lifts to a rectangle depending differentiably on $t$ in $\mathbb{H}^{3}$.

In $[5, \S 10]$, we showed that the restriction of $f_{t}$ to each component of $S-\lambda_{t}$ depends differentiably on $t$. Namely, if we lift $f_{t}$ to a pleated surface $\widetilde{f}_{t}: \widetilde{S} \rightarrow \mathbb{H}^{3}$ defined on the universal covering $\widetilde{S}$, then for every component $P$ of the complement of the preimage $\tilde{\lambda}$ of $\lambda$ in $\widetilde{S}$, the ideal triangle $\widetilde{f}_{t}\left(P_{t}\right)$ in $\mathbb{H}^{3}$ depends differentiably on $t$, where $P_{t}$ denotes the corresponding component of $\widetilde{S}-\widetilde{\lambda}_{t}$. (Strictly speaking, we proved this property only if we replace the isometric identification $M_{t} \cong \mathbb{H}^{3} / \rho_{t}(\Gamma)$ by another identification $M_{t} \cong \mathbb{H}^{3} / \rho_{t}^{\prime}(\Gamma)$ where there exists $A_{t} \in \mathrm{Isom}^{+}\left(\mathbb{H}^{3}\right)$ such that $\rho_{t}^{\prime}(\gamma)=A_{t}^{-1} \rho_{t}(\gamma) A_{t}$ for every $\gamma \in \Gamma$.

For every $\gamma \in \pi_{1}(S) \subset \Gamma$, the fact that $\widetilde{f}_{t}\left(P_{t}\right)$ and $\widetilde{f}_{t}\left(\gamma P_{t}\right)$ depend differentiably on $t$ for an arbitrary component $P$ of $\widetilde{S}-\widetilde{\lambda}$ shows that $\rho_{t}^{\prime}(\gamma)$ depends differentiably on $t$. Looking at the fixed points of the isometry groups $\rho_{t}\left(\pi_{1}(S)\right)$ and $\rho_{t}^{\prime}\left(\pi_{1}(S)\right)$, we conclude that $A_{t}$ depends differentiably on $t$. Using the identification $\mathbb{H}^{3} / \rho_{t}(\Gamma) \cong \mathbb{H}^{3} / \rho_{t}^{\prime}(\Gamma)$ induced by $A_{t}$, we can therefore assume that $\rho_{t}^{\prime}=\rho_{t}$.)

[5] also shows that the pull back metric $m_{t}$ on $S$ depends differentiably on $t$. Again, $[5, \S 5]$ provides a representation $\sigma_{t}: \pi_{1}(S) \rightarrow$ Isom ${ }^{+}\left(\mathbb{H}^{2}\right)$ depending differentiably on $t$ and an isometric identification $\varphi_{t}:\left(S, m_{t}\right) \rightarrow \mathbb{H}^{2} / \sigma_{t}\left(\pi_{1}(S)\right)$ such that, for every component $P$ of $\widetilde{S}-\widetilde{\lambda}$, the image $\widetilde{\varphi}_{t}\left(P_{t}\right)$ of the corresponding component $P_{t}$ of $\widetilde{S}-\widetilde{\lambda}_{t}$ under a lift $\widetilde{\varphi}_{t}: \widetilde{S} \rightarrow \mathbb{H}^{2}$ is an ideal triangle which varies differentiably with $t$. We assumed that the vertices of the rectangles $R_{i}^{(t)}$ depend differentiably on $t$ for the metric $m_{t}$, namely that, if we lift $R_{i}^{(t)}$ to $\widetilde{R}_{i}^{(t)} \subset \widetilde{S}$, the vertices of $\widetilde{\varphi}_{t}\left(\widetilde{R}_{i}^{(t)}\right) \subset \mathbb{H}^{2}$ depend differentiably on $t$. If $P_{t}$ is the component of $\widetilde{S}-\widetilde{\lambda}_{t}$ that contains the lift $\widetilde{R}_{t} \subset \widetilde{R}_{i}^{(t)}$ of $R_{t}$, and we $m_{t}$-isometrically identify $P_{t}$ with a fixed ideal triangle, then $\widetilde{R}_{t}=\widetilde{\varphi}_{t}^{-1}\left(\widetilde{\varphi}_{t}\left(\widetilde{R}_{i}^{(t)}\right) \cap \widetilde{\varphi}_{t}\left(P_{t}\right)\right)$ depends differentiably on $t$ in this fixed ideal triangle.

Because $\tilde{f}_{t}\left(P_{t}\right)$ depends differentiably on $t$, we conclude that $\widetilde{f}_{t}\left(\widetilde{R}_{t}\right)$ depends differentiably on $t$ in $\mathbb{H}^{3}$, so that $f_{t}\left(R_{t}\right)$ depends differentiably on $t$ in $M_{t}$. q.e.d.

Lemma 4 shows that each of the pyramids and tetrahedra of the decomposition of $H_{t}\left(R_{i}^{(t)} \times[0,1]\right)$ varies differentiably with $t$. 
We need to precise Lemma 4, using the estimates of [5]. When $R_{t}$ does not contain one of the sides of $R_{i}^{(t)}$, the two leaves of $\lambda_{t}$ that it touches follow each other for a while, crossing the same rectangles $R_{j}^{(t)}$. However, in some direction, they must diverge and cross different $R_{j}^{(t)}$ after a while, since they would otherwise stay within bounded distance of each other and therefore be equal. Let the divergence radius $r\left(R_{t}\right) \geqslant 1$ be the number of $R_{j}^{(t)}$ which they cross in common before diverging. By convention, $r\left(R_{t}\right)=1$ when $R_{t}$ contains one of the sides of $R_{i}^{(t)}$.

Lemma 5. With the data of Lemma 4, the derivative $\dot{a}_{t}$ of each vertex $a_{t}$ of $f_{t}\left(R_{t}\right)$ with respect to $t$ is an $O\left(r\left(R_{t}\right)\right)=O\left(r\left(R_{0}\right)\right)$. In addition, for any two vertices $a_{t}, b_{t}$, the distance between the vectors $\dot{a}_{t}$ and $\dot{b}_{t}$ is an $O\left(d\left(a_{t}, b_{t}\right) r\left(R_{t}\right)\right)$.

Proof. To give a sense to this statement, we need to choose a lift of $f_{t}\left(R_{t}\right) \subset M_{t} \cong \mathbb{H}^{3} / \rho_{t}(\Gamma)$ to $\mathbb{H}^{3}$, as in Lemma 4. For this, we first choose a lift of $f_{t}\left(R_{i}^{(t)}\right)$ to $\mathbb{H}^{3}$, and then restrict it to a lift of each $f_{t}\left(R_{t}\right)$. The statement of Lemma 5 implicitly assumes that the lifts of the $f_{t}\left(R_{t}\right)$ are chosen in such a consistent way. The constants hidden in the symbols $O\left(\right.$ ) will then depend on the choice of the lift of $f_{t}\left(R_{i}^{(t)}\right)$, but not on the components $R_{t}$.

Let us use the notation of the proof of Lemma 4 . In $[5, \S 10]$, we give explicit formulas expressing the restriction of $\widetilde{f}_{t+h}$ to each component of $\widetilde{S}-\widetilde{\lambda}_{t+h}$ as a limit of rotation-translations along geodesics of $\widetilde{f}_{t}\left(\widetilde{\lambda}_{t}\right)$. In addition, we show that the convergence is holomorphic, so that we can differentiate in the limit. Differentiating with respect to $h$ and applying to the derivative the estimates of $[5, \S 5]$ and $[5$, Lemma 6$]$, it easily follows that the derivative of the ideal triangle $\tilde{f}_{t}\left(P_{t}\right)$ is an $O\left(r\left(R_{t}\right)\right)$. Similarly, the derivative of $\widetilde{\varphi}_{t}\left(P_{t}\right)$ is also an $O\left(r\left(R_{t}\right)\right)$. The first statement of Lemma 5 immediately follows.

For the second statement, we can restrict attention to the case where $a_{t}$ and $b_{t}$ are on the same side of $f_{t}\left(R_{i}^{(t)}\right)$; indeed, $d\left(a_{t}, b_{t}\right)$ is otherwise bounded away from 0 , and the result is trivial. Then, $a_{t}$ and $b_{t}$ are the images under $\tilde{f}_{t} \circ\left(\widetilde{\varphi}_{t \mid P_{t}}\right)^{-1}$ of the intersection points of $\widetilde{\varphi}_{t}\left(\partial P_{t}\right)$ with an arc $k_{t}$ in $\mathbb{H}^{2}$ which varies differentiably with $t$ (and corresponds to a side of $\left.R_{i}^{(t)}\right)$. Because $\widetilde{f}_{t \mid P_{t}}$ and $\widetilde{\varphi}_{t \mid P_{t}}$ are isometries, their differentials are uniformly Lipschitz, and the $\mathrm{C}^{1}$-norm of their derivatives with respect to $t$ is an $O\left(r\left(R_{t}\right)\right)$ by the previous estimate. The second statement of Lemma 5 then follows from the chain rule. q.e.d. 


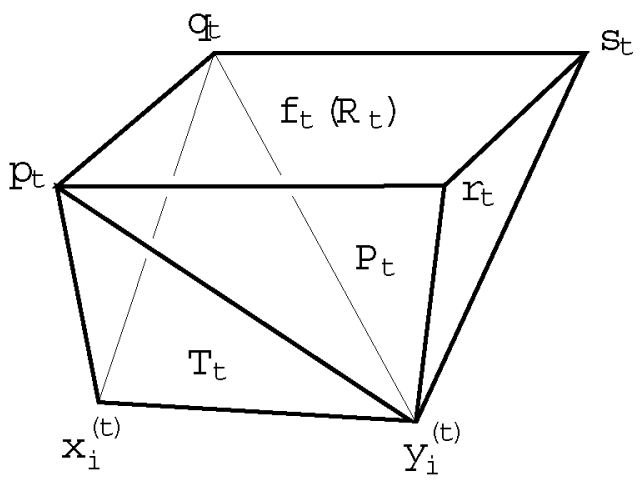

Figure 2

To apply the Schläfli formula to these pyramids and tetrahedra, we need to make sure that their faces are not collapsed to arcs or points (otherwise, dihedral angles do not make sense). This means that for every $t$ the points $x_{i}^{(t)}$ and $y_{i}^{(t)}$ avoid a countable union of geodesic arcs of bounded length in $M_{t}$. By Lemma 4, these geodesic arcs depend differentiably on $t$. As a consequence, their lifts sweep a domain of Lebesgue measure 0 in $\mathbb{H}^{3}$. We can therefore choose the polyhedral maps $g_{t}$ generic enough so that the pyramids and tetrahedra are never degenerate. Actually, we only need this property for the sake of the exposition, since the estimate of Lemma 6 below would enable us to deal with degenerate pyramids and tetrahedra as well.

Also, the image $f_{t}\left(\lambda_{t}\right)$ of the pleating locus has Hausdorff dimension 1 [2], and varies continuously with $t$; see $[21, \S 8][8, \S 5]$ or [5]. Therefore, we can arrange that the points $x_{i}^{(t)}$ and $y_{i}^{(t)}$ stay at distance bounded away from 0 from $f_{t}\left(\lambda_{t}\right)$.

Let $R_{t}$ be a component of $R_{i}^{(t)}-\lambda_{t}$, and let $p_{t}, q_{t}, r_{t}, s_{t}$ be the vertices of the totally geodesic rectangle $f_{t}\left(R_{t}\right)$ where, for the identification $R_{i}^{(t)} \cong[a, b] \times[c, d]$, the points $p_{t}, q_{t}$ occur in this order in $f_{t}([a, b] \times\{c\})$ and the points $r_{t}, s_{t}$ occur in this order in $f_{t}([a, b] \times\{d\})$. Then, the pyramid $P_{t}$ associated to $R_{t}$ has vertices $p_{t}, q_{t}, r_{t}, s_{t}$ and $y_{i}^{(t)}$. See Figure 2.

The Schläfli formula gives that $d \operatorname{vol}\left(P_{t}\right) / d t$ is the sum of the terms

$$
\frac{1}{2} l\left(p_{t} q_{t}\right) \frac{d}{d t} \theta_{P_{t}}\left(p_{t} q_{t}\right)
$$




$$
\begin{gathered}
\frac{1}{2} l\left(r_{t} s_{t}\right) \frac{d}{d t} \theta_{P_{t}}\left(r_{t} s_{t}\right), \\
\frac{1}{2}\left(l\left(p_{t} y_{i}^{(t)}\right) \frac{d}{d t} \theta_{P_{t}}\left(p_{t} y_{i}^{(t)}\right)+l\left(q_{t} y_{i}^{(t)}\right) \frac{d}{d t} \theta_{P_{t}}\left(q_{t} y_{i}^{(t)}\right)\right), \\
\frac{1}{2}\left(l\left(r_{t} y_{i}^{(t)}\right) \frac{d}{d t} \theta_{P_{t}}\left(r_{t} y_{i}^{(t)}\right)+l\left(s_{t} y_{i}^{(t)}\right) \frac{d}{d t} \theta_{P_{t}}\left(s_{t} y_{i}^{(t)}\right)\right),
\end{gathered}
$$

and

$$
\frac{1}{2}\left(l\left(p_{t} r_{t}\right) \frac{d}{d t} \theta_{P_{t}}\left(p_{t} r_{t}\right)+l\left(s_{t} q_{t}\right) \frac{d}{d t} \theta_{P_{t}}\left(s_{t} q_{t}\right)\right)
$$

where $l()$ denotes the length of the edge indicated, and $\theta_{P_{t}}()$ is the external dihedral angle of the boundary $\partial P_{t}$ at the edge indicated. For this, we orient $\partial P_{t}$ so that the restriction $f_{t \mid P_{t}}: R_{t} \rightarrow f_{t}\left(R_{t}\right) \subset \partial P_{t}$ is orientation-preserving and we orient $P_{t}$ accordingly (so that $\operatorname{vol}\left(P_{t}\right)$ may be negative).

The grouping of the terms is here important, because it will guarantee the convergence of the series when we sum over all components $R_{t}$ of $R_{i}^{(t)}-\lambda_{t}$. It is not hard to see that this sum will not converge if we do not use this grouping.

We first sum the terms of type (4). By the second part of Lemma 5 , $d \theta_{P_{t}}\left(p_{t} q_{t}\right) / d t=O\left(r\left(R_{0}\right)\right)$. Also, there is a constant $A>0$, depending only on the length of the components of $\lambda_{t} \cap R_{i}^{(t)}$ (and therefore uniform in $t$ ), such that the leaves of $\lambda_{t}$ passing through $p_{t}$ and $q_{t}$ stay at uniformly bounded distance from each other over a length of at least $\operatorname{Ar}\left(R_{t}\right)=\operatorname{Ar}\left(R_{0}\right)$; since the metric $m_{t}$ is hyperbolic, it follows that $l\left(p_{t} q_{t}\right)=O\left(e^{-A r\left(R_{0}\right)}\right)$. Finally, for $r \geqslant 1$ large enough, the number of components $R_{0}$ of $R_{i}^{(0)}-\lambda_{0}$ such that $r\left(R_{0}\right)=r$ is uniformly bounded by the number of spikes of $S-\lambda_{0}$. It follows that, as we sum over all components $R_{t}$ of $R_{i}^{(t)}-\lambda_{t}$, the series

$$
\frac{1}{2} \sum_{R_{t}} l\left(p_{t} q_{t}\right) \frac{d}{d t} \theta_{P_{t}}\left(p_{t} q_{t}\right)
$$

is convergent.

The same arguments show the convergence of the sum of the terms of type (5), namely of

$$
\frac{1}{2} \sum_{R_{t}} l\left(r_{t} s_{t}\right) \frac{d}{d t} \theta_{P_{t}}\left(r_{t} s_{t}\right)
$$




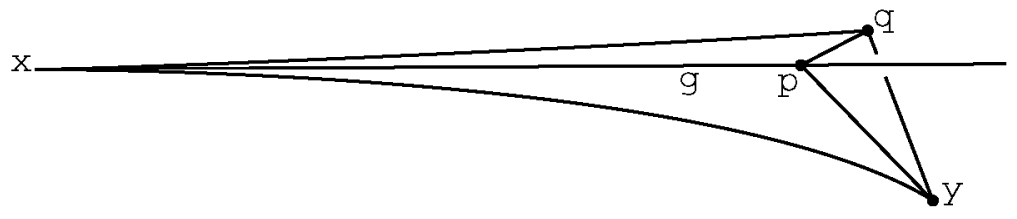

Figure 3

To show the convergence of the sum of the terms of type (6), we first estimate the quantity

$$
\frac{d}{d t} \theta_{P_{t}}\left(p_{t} y_{i}^{(t)}\right)+\frac{d}{d t} \theta_{P_{t}}\left(q_{t} y_{i}^{(t)}\right)
$$

For this, choose an isometric embedding $\varphi_{t}: P_{t} \rightarrow \mathbb{H}^{3}$ such that $\varphi_{t}\left(f_{t}\left(R_{t}\right)\right)$ is contained in $\mathbb{H}^{2} \subset \mathbb{H}^{3}, \varphi_{t}\left(p_{t} r_{t}\right)$ is contained in a fixed geodesic $g$ of $\mathbb{H}^{2}$, and $\varphi_{t}\left(p_{t}\right)$ is a fixed point $p \in g$. Since the two leaves of $\lambda_{t}$ touching $R_{t}$ are asymptotic, the geodesic of $\mathbb{H}^{2}$ that contains $\varphi_{t}\left(q_{t}\right)$ and $\varphi_{t}\left(s_{t}\right)$ has an end point $x$ at infinity in common with $g$. We can now consider the tetrahedron $T$ with vertices $p=\varphi_{t}\left(p_{t}\right), q=\varphi_{t}\left(q_{t}\right)$, $y=\varphi_{t}\left(y_{i}^{(t)}\right)$ and $x$; see Figure 3 .

Lemma 6. Let $g$ be a geodesic of $\mathbb{H}^{2} \subset \mathbb{H}^{3}$, let $p$ be a fixed point of $g$, and let $x$ be one of the end points of $g$ on the circle at infinity $\partial_{\infty} \mathbb{H}^{2}$. Given two constants $A>0$ and $B>0$, consider two points $q \in \mathbb{H}^{2}$ and $y \in \mathbb{H}^{3}$ such that the distances from $y$ to $p$ and from $p$ to $q$ are at most $A$, and such that the distance from $y$ to $g$ and to the geodesic of $\mathbb{H}^{2}$ containing $q$ and $x$ is at least $B$. Finally, in the tetrahedron $T$ of vertices $p, q, x, y$, let $\Theta(q, y)$ denote the sum of the internal dihedral angles of $T$ along the edges py and $q y$. Then, $\Theta(q, y)$ is a differentiable function of $q$ and $y$. In addition, if $q$ varies with velocity $\dot{q}$ and $y$ varies with velocity $\dot{y}$, the derivative $\dot{\Theta}(q, y)$ of $\Theta(q, y)$ is an $O(\|\dot{q}\|+d(p, q)\|\dot{y}\|)$, where the constant hidden in the symbol $O($ ) depends only on the constants $A$ and $B$.

Proof. Note that our definition of $\Theta(q, y)$ does not make sense when the tetrahedron $T$ is degenerate, namely when the three points $p$, $q$ and $y$ are on the same geodesic (since $B>0$, the triangles $x p y$ and $x q y$ cannot be degenerate). We first extend it to this case, by using a different point of view.

At the point $y$, consider the unit vectors $v_{p}, v_{q}, v_{x}$ pointing in the direction of $p, q, x$, respectively. These vectors draw a triangle $v_{x} v_{p} v_{q}$ 
on the visual sphere at $y$. Then, $\Theta(q, y)$ is the sum of the angles of this spherical triangle at $v_{p}$ and $v_{q}$, when these angles make sense. By the Gauss formula, $\Theta(q, y)$ is therefore equal to $\pi$ plus the area of the spherical triangle $v_{x} v_{p} v_{q}$ minus the angle of the triangle at $v_{x}$. Since $y$ stays away from $g$ and the geodesic containing $x$ and $q$, the edges $v_{x} v_{p}$ and $v_{x} v_{q}$ of the triangle are never reduced to a point. This formula for $\Theta(q, y)$ consequently makes sense for every $q, y$ satisfying the conditions of the Lemma, and shows that $\Theta(q, y)$ is an infinitely differentiable function of $q$ and $y$.

The positions allowed by the conditions of Lemma 6 for the point $(q, y)$ form a compact subset of $\mathbb{H}^{2} \times \mathbb{H}^{3}$. If we let $q$ vary with velocity $\dot{q}$ while $y$ stays fixed, the corresponding derivative $\dot{\Theta}(q, y)$ of $\Theta(q, y)$ depends linearly on $\dot{q}$ and continuously on $(q, y)$. It follows that $\dot{\Theta}(q, y)$ is an $O(\|\dot{q}\|)$ in this case.

If we let $y$ vary with velocity $\dot{y}$ while $q$ stays fixed, the corresponding derivative $\dot{\Theta}(q, y)$ depends linearly on $\dot{y}$ and differentiably on $(q, y)$. In addition, if $q$ is equal to $p, \Theta(q, y)$ is constantly equal to $\pi$ under such a variation, so that $\dot{\Theta}(p, y)$ is equal to 0 . The same compactness arguments as above now shows that $\dot{\Theta}(q, y)$ is an $O(d(p, q)\|\dot{y}\|)$ in this case.

The case of a general variation follows from these two cases by linearity of the differential of $\Theta$. q.e.d.

We can apply Lemma 6 to the tetrahedron $T$ with vertices $p=$ $\varphi_{t}\left(p_{t}\right), q=\varphi_{t}\left(q_{t}\right), y=\varphi_{t}\left(y_{i}^{(t)}\right)$ and $x$, where $x$ is the end point at infinity that is common to the geodesic containing $\varphi_{t}\left(p_{t}\right)$ and $\varphi_{t}\left(r_{t}\right)$ and the geodesic containing $\varphi_{t}\left(q_{t}\right)$ and $\varphi_{t}\left(s_{t}\right)$. In the pyramid $P_{t}$, $\theta_{P_{t}}\left(p_{t} y_{i}^{(t)}\right)+\theta_{P_{t}}\left(q_{t} y_{i}^{(t)}\right)$ is equal to $\Theta(q, y)$ or to $2 \pi-\Theta(q, y)$, according as $x$ sits with respect to $\varphi_{t}\left(p_{t}\right)$ and $\varphi_{t}\left(r_{t}\right)$. When we differentiate with respect to $t$, the variation $\dot{p}$ of $p$ is equal to 0 , since $p$ is constant. By Lemma 5 , the variation $\dot{q}$ of $q$ is an $O\left(d\left(p_{t}, q_{t}\right) r\left(R_{0}\right)\right)$, and the variation $\dot{y}$ of $y$ is an $O\left(r\left(R_{0}\right)\right)$. Since there is a constant $A>0$ such that $d\left(p_{t}, q_{t}\right)=O\left(e^{-A r\left(R_{0}\right)}\right)$ and the distance from $y_{i}^{(t)}$ to $f_{t}\left(\lambda_{t}\right)$ is bounded away from 0 , Lemma 6 shows that

$$
\frac{d}{d t} \theta_{P_{t}}\left(p_{t} y_{i}^{(t)}\right)+\frac{d}{d t} \theta_{P_{t}}\left(q_{t} y_{i}^{(t)}\right)=O\left(r\left(R_{0}\right) e^{-A r\left(R_{0}\right)}\right) .
$$

The lengths $l\left(p_{t} y_{i}^{(t)}\right)$ and $l\left(q_{t} y_{i}^{(t)}\right)$ are uniformly bounded, and

$$
l\left(p_{t} y_{i}^{(t)}\right)-l\left(q_{t} y_{i}^{(t)}\right)=O\left(l\left(p_{t} q_{t}\right)\right)=O\left(e^{-A r\left(R_{0}\right)}\right) .
$$


Since $d \theta_{P_{t}}\left(q_{t} y_{i}^{(t)}\right) / d t=O\left(r\left(R_{0}\right)\right)$ by Lemma 5 , it follows that

$$
\begin{aligned}
l\left(p_{t} y_{i}^{(t)}\right) \frac{d}{d t} \theta_{P_{t}}\left(p_{t} y_{i}^{(t)}\right)+ & l\left(q_{t} y_{i}^{(t)}\right) \frac{d}{d t} \theta_{P_{t}}\left(q_{t} y_{i}^{(t)}\right) \\
& =O\left(r\left(R_{0}\right) e^{-A r\left(R_{0}\right)}\right) .
\end{aligned}
$$

Since, for every $r \geqslant 1$, the number of components $R_{0}$ of $R_{i}^{(0)}-\lambda_{0}$ such that $r\left(R_{0}\right)=r$ is uniformly bounded, (12) guarantees the convergence of the series

$$
\frac{1}{2} \sum_{R_{t}}\left(l\left(p_{t} y_{i}^{(t)}\right) \frac{d}{d t} \theta_{P_{t}}\left(p_{t} y_{i}^{(t)}\right)+l\left(q_{t} y_{i}^{(t)}\right) \frac{d}{d t} \theta_{P_{t}}\left(q_{t} y_{i}^{(t)}\right)\right)
$$

as we sum over all components $R_{t}$ of $R_{i}^{(t)}-\lambda_{t}$.

The convergence of the sums

$$
\frac{1}{2} \sum_{R_{t}}\left(l\left(r_{t} y_{i}^{(t)}\right) \frac{d}{d t} \theta_{P_{t}}\left(r_{t} y_{i}^{(t)}\right)+l\left(s_{t} y_{i}^{(t)}\right) \frac{d}{d t} \theta_{P_{t}}\left(s_{t} y_{i}^{(t)}\right)\right),
$$

and

$$
\frac{1}{2} \sum_{R_{t}}\left(l\left(p_{t} r_{t}\right) \frac{d}{d t} \theta_{P_{t}}\left(p_{t} r_{t}\right)+l\left(s_{t} q_{t}\right) \frac{d}{d t} \theta_{P_{t}}\left(s_{t} q_{t}\right)\right)
$$

is proved by arguments which are, identical to the above one for (14), and very similar for (15).

Now, consider the tetrahedron $T_{t}$, with vertices $p_{t}, q_{t}, x_{i}^{(t)}, y_{i}^{(t)}$, which is also associated to $R_{t}$. Orient the boundary $\partial T_{t}$ so that the orientation induced on the triangle $p_{t} q_{t} y_{i}^{(t)}$ is opposite to the orientation induced by the boundary $\partial P_{t}$, and orient $T_{t}$ accordingly. The Schläfli formula expresses $d \mathrm{vol}\left(T_{t}\right) / d t$ as a sum of terms corresponding to its edges. Summing over all components $R_{t}$ of $R_{i}^{(t)}-\lambda_{t}$, we obtain the following four sums, whose convergence is proved by arguments similar to the ones used for $P_{t}$.

$$
\begin{gathered}
\frac{1}{2} \sum_{R_{t}} l\left(p_{t} q_{t}\right) \frac{d}{d t} \theta_{T_{t}}\left(p_{t} q_{t}\right), \\
\frac{1}{2} \sum_{R_{t}} l\left(x_{i}^{(t)} y_{i}^{(t)}\right) \frac{d}{d t} \theta_{T_{t}}\left(x_{i}^{(t)} y_{i}^{(t)}\right),
\end{gathered}
$$




$$
\frac{1}{2} \sum_{R_{t}}\left(l\left(p_{t} x_{i}^{(t)}\right) \frac{d}{d t} \theta_{T_{t}}\left(p_{t} x_{i}^{(t)}\right)+l\left(q_{t} x_{i}^{(t)}\right) \frac{d}{d t} \theta_{T_{t}}\left(q_{t} x_{i}^{(t)}\right)\right)
$$

and

$$
\frac{1}{2} \sum_{R_{t}}\left(l\left(p_{t} y_{i}^{(t)}\right) \frac{d}{d t} \theta_{T_{t}}\left(p_{t} y_{i}^{(t)}\right)+l\left(q_{t} y_{i}^{(t)}\right) \frac{d}{d t} \theta_{T_{t}}\left(q_{t} y_{i}^{(t)}\right)\right)
$$

converge, by arguments similar to the ones used for $P_{t}$.

Since the convergence of all these sums is uniform in $t$, we conclude that the volume of $H_{t}\left(R_{i}^{(t)} \times[0,1]\right)$ is differentiable in $t$, and that

$$
\frac{d}{d t} \operatorname{vol}\left(H_{t}\left(R_{i}^{(t)} \times[0,1]\right)\right)=\frac{d}{d t} \sum_{R_{t}} \operatorname{vol}\left(P_{t}\right)+\frac{d}{d t} \sum_{R_{t}} \operatorname{vol}\left(T_{t}\right)
$$

is equal to the sum of all terms $(9-10),(13-15)$ and (16-19) as $R_{t}$ ranges over all components of $R_{i}^{(t)}-\lambda_{t}$. (We are here using an abuse of notation, where $H_{t}\left(R_{i}^{(t)} \times[0,1]\right)$ represents the chain defined by restriction of $H_{t}$ to $R_{i}^{(t)} \times[0,1]$ and not the image of this map. In particular, the volume is computed by taking into account the sign of the Jacobian of $H_{t}$, and may very well be negative. We will use the same abuse of notation below when considering the boundary of this chain.)

The term (17) is particularly simple. Indeed, consider the corners $p_{i}^{(t)}=f_{t}(a, c), q_{i}^{(t)}=f_{t}(b, c), r_{i}^{(t)}=f_{t}(a, d), s_{i}^{(t)}=f_{t}(b, d)$ of the image of the rectangle $R_{i}^{(t)} \cong[a, b] \times[c, d]$ under $f_{t}$, as in Figure 1 . Then, the $\operatorname{sum}(17)$ is equal to

$$
\frac{1}{2} l\left(x_{i}^{(t)} y_{i}^{(t)}\right) \frac{d}{d t} \theta_{H_{t}\left(R_{i}^{(t)} \times[0,1]\right)}\left(x_{i}^{(t)} y_{i}^{(t)}\right)
$$

where $\theta_{H_{t}\left(R_{i}^{(t)} \times[0,1]\right)}\left(x_{i}^{(t)} y_{i}^{(t)}\right)$ is the external dihedral angle between the triangles $x_{i}^{(t)} y_{i}^{(t)} p_{i}^{(t)}$ and $x_{i}^{(t)} y_{i}^{(t)} q_{i}^{(t)}$. Indeed, this follows from the fact that

(21) $\pi-\theta_{H_{t}\left(R_{i}^{(t)} \times[0,1]\right)}\left(x_{i}^{(t)} y_{i}^{(t)}\right)=\sum_{R_{t}}\left(\pi-\theta_{T_{t}}\left(x_{i}^{(t)} y_{i}^{(t)} p_{t}, y_{i}^{(t)} x_{i}^{(t)} q_{t}\right)\right)$

in $\mathbb{R} / 2 \pi \mathbb{Z}$ (because $f_{t}\left(([a, b] \times\{c\}) \cap \lambda_{t}\right)$ has 1 -dimensional measure 0 ) and that the convergence of the sum (17) is uniform in $t$. 
It turns out that the terms (13) and (19) almost cancel out. Indeed, they both involve edges of the form $p_{t} y_{i}^{(t)}$ and $q_{t} y_{i}^{(t)}$. In general, the contributions to (13) and (19) of each individual edge $p_{t} y_{i}^{(t)}$ or $q_{t} y_{i}^{(t)}$ do not add up to 0 . However, we will show that only four terms remain when we sum these contributions over all rectangles $R_{t}$. This will require the consideration of the bending cocycle of a certain pleated fan.

Consider the closure $P_{i}^{(t)} \subset H_{t}\left(R_{i}^{(t)} \times[0,1]\right)$ of the union of the pyramids $P_{t}$. It is partially bounded by the joint $F_{i}^{(t)}$ of the point $y_{i}^{(t)}$ and the $\operatorname{arc} f_{t}([a, b] \times\{c\})$. This $F_{i}^{(t)}$ is a pleated fan whose pleating locus is the joint $\mu_{i}^{(t)}$ of $y_{i}^{(t)}$ and $f_{t}\left(([a, b] \times\{c\}) \cap \lambda_{t}\right)$. We orient $F_{i}^{(t)}$ so that the boundary orientation it induces on $f_{t}\left(([a, b] \times\{c\}) \cap \lambda_{t}\right)$ coincides with the one coming from the natural orientation of $[a, b]$. Using the methods of $[5, \S 7]$, we can measure the bending of $F_{i}^{(t)}$ along $\mu_{i}^{(t)}$ by an $\mathbb{R} / 2 \pi \mathbb{Z}$-valued transverse cocycle $\beta_{t}$ for $\mu_{i}^{(t)}$; the crucial property here is that the curve $f_{t}([a, b] \times\{c\})$ is rectifiable. We now interpret the quantity (13) as, essentially, the length of the derivative of this bending cocycle.

Lemma 7. As we differentiate in $t$, the bending cocycle $\beta_{t}$ of the pleated fan $F_{i}^{(t)}$ admits a derivative $\dot{\beta}_{t}$, which is an $\mathbb{R}$-valued transverse cocycle for the pleating locus $\mu_{i}^{(t)}$. In addition, $\dot{\beta}_{t}$ has a well defined length $l\left(\dot{\beta}_{t}\right)$ in $M_{t}$, and the quantity (13) is equal to

$$
\frac{1}{2} l\left(\dot{\beta}_{t}\right)+\frac{1}{2} l\left(p_{i}^{(t)} y_{i}^{(t)}\right) \frac{d}{d t} \theta_{P_{i}^{(t)}}\left(p_{i}^{(t)} y_{i}^{(t)}\right)+\frac{1}{2} l\left(q_{i}^{(t)} y_{i}^{(t)}\right) \frac{d}{d t} \theta_{P_{i}^{(t)}}\left(q_{i}^{(t)} y_{i}^{(t)}\right)
$$

Proof. For every $x \in \lambda_{t} \cap R_{i}^{(t)}$, consider the tangent plane at $f_{t}(x) \in M_{t}$ that is tangent to the geodesic arc $f_{t}(x) y_{i}^{(t)}$ and to the image under $f_{t}$ of the leaf of $\lambda_{t}$ containing $x$; this plane is a Lipschitz function of $x$. Consequently, the expression of the bending cocycle given by Lemma 36 of [5] shows that, for every arc $k$ in $[a, b] \times\{c\}$ whose end points are disjoint from $\lambda_{t}$,

$$
\begin{aligned}
\beta_{t}(k)= & \sum_{R_{t} \cap k \neq \emptyset}\left(\theta_{P_{t}}\left(p_{t} y_{i}^{(t)}\right)-\pi+\theta_{P_{t}}\left(q_{t} y_{i}^{(t)}\right)\right) \\
& -\theta_{P_{t}^{-}}\left(p_{t}^{-} y_{i}^{(t)}\right)+\pi-\theta_{P_{t}^{+}}\left(q_{t}^{+} y_{i}^{(t)}\right),
\end{aligned}
$$

where the sum is taken over all components $R_{t}$ of $R_{i}^{(t)}-\lambda_{t}$ that meet $k$, and $P_{t}^{ \pm}, p_{t}^{ \pm}, q_{t}^{ \pm}$denote the pyramid and vertices associated to $f_{t}\left(R_{t}^{ \pm}\right)$ 
by the usual labelling conventions, $R_{t}^{+}$and $R_{t}^{-}$being the components of $R_{i}^{(t)}-\lambda_{t}$ that respectively contain the positive and negative end point of $k$. From Lemma 4 and (11), we conclude that $\beta_{t}(k)$ has a derivative $\dot{\beta}_{t}(k) \in \mathbb{R}$ with respect to $t$, given by

$$
\begin{aligned}
\dot{\beta}_{t}(k)= & \sum_{R_{t} \cap k \neq \emptyset}\left(\frac{d}{d t} \theta_{P_{t}}\left(p_{t} y_{i}^{(t)}\right)+\frac{d}{d t} \theta_{P_{t}}\left(q_{t} y_{i}^{(t)}\right)\right) \\
& -\frac{d}{d t} \theta_{P_{t}^{-}}\left(p_{t}^{-} y_{i}^{(t)}\right)-\frac{d}{d t} \theta_{P_{t}^{+}}\left(q_{t}^{+} y_{i}^{(t)}\right)
\end{aligned}
$$

Since the finite additivity with respect to $k$ is immediate, this defines a transverse $\mathbb{R}$-valued cocycle $\dot{\beta}_{t}$ for the pleating locus $\mu_{i}^{(t)}$ of the pleated fan $F_{i}^{(t)}$.

In [3], we showed how an $\mathbb{R}$-valued transverse cocycle for a geodesic lamination $\mu$ on $S$ defines a transverse Hölder distribution for $\mu$. However, this construction depended in a crucial way on some global properties of $\mu$. Associating a transverse Hölder distribution for $\mu_{i}^{(t)}$ to the transverse cocycle $\dot{\beta}_{t}$ is therefore not automatic. However, by (24), (11) and Lemma $5, \dot{\beta}_{t}(k)=O\left(r\left(R_{t}^{+}\right)\right)+O\left(r\left(R_{t}^{-}\right)\right)$for every $k$, and $d\left(p_{t}, q_{t}\right)=O\left(e^{-\operatorname{Ar}\left(R_{t}\right)}\right)$ for every $R_{t}$, with the usual notation. Since, for every $r \geqslant 0$, the number of $R_{t}$ with $r\left(R_{t}\right)=r$ is uniformly bounded, this is exactly what we need to use the techniques of [3] and associate to $\dot{\beta}_{t}$ a transverse Hölder distribution for $\mu_{i}^{(t)}$; compare (25) below. In particular, we can integrate with respect to this distribution the length of the leaves of $\mu_{i}^{(t)}$, which defines the length $l\left(\dot{\beta}_{t}\right)$.

Theorem 11 of [3] provides an explicit expression for the transverse distribution $\dot{\beta}_{t}$, which gives

$$
\begin{aligned}
l\left(\dot{\beta}_{t}\right)= & \sum_{R_{t}} \dot{\beta}_{t}\left(k\left(R_{t}\right)\right)\left(l\left(p_{t} y_{i}^{(t)}\right)-l\left(q_{t} y_{i}^{(t)}\right)\right) \\
& +\dot{\beta}_{t}\left(f_{t}([a, b] \times\{c\})\right) l\left(q_{i}^{(t)} y_{i}^{(t)}\right),
\end{aligned}
$$

where $k\left(R_{t}\right)$ denotes the $\operatorname{arc}$ in $[a, b] \times\{c\}$ that joins $(a, c)$ to an arbitrary point in the interior of $([a, b] \times\{c\}) \cap R_{t}$. Also, the Gap Lemma of [3] shows that

$$
l\left(q_{t} y_{i}^{(t)}\right)=\sum_{R_{t}^{\prime} \cap k\left(R_{t}\right)=\emptyset}\left(l\left(p_{t}^{\prime} y_{i}^{(t)}\right)-l\left(q_{t}^{\prime} y_{i}^{(t)}\right)\right)+l\left(q_{i}^{(t)} y_{i}^{(t)}\right)
$$

where the sum is over those components $R_{t}^{\prime}$ of $R_{i}^{(t)}-\lambda_{t}$ which do not meet the arc $k\left(R_{t}\right)$, and $p_{t}^{\prime}, q_{t}^{\prime}, r_{t}^{\prime}, s_{t}^{\prime}$ denote the vertices of $f_{t}\left(R_{t}^{\prime}\right)$ 
with the usual labelling conventions. Combining (24), (25), (26) and rearranging terms, we conclude that

$$
\begin{aligned}
l\left(\dot{\beta}_{t}\right)= & \sum_{R_{t}}\left(l\left(p_{t} y_{i}^{(t)}\right) \frac{d}{d t} \theta_{P_{t}}\left(p_{t} y_{i}^{(t)}\right)+l\left(q_{t} y_{i}^{(t)}\right) \frac{d}{d t} \theta_{P_{t}}\left(q_{t} y_{i}^{(t)}\right)\right) \\
& -l\left(p_{i}^{(t)} y_{i}^{(t)}\right) \frac{d}{d t} \theta_{P_{i}^{(t)}}\left(p_{i}^{(t)} y_{i}^{(t)}\right)-l\left(q_{i}^{(t)} y_{i}^{(t)}\right) \frac{d}{d t} \theta_{P_{i}^{(t)}}\left(q_{i}^{(t)} y_{i}^{(t)}\right) .
\end{aligned}
$$

This completes the proof of Lemma 7 . q.e.d.

Similarly, if $T_{i}^{(t)}$ denotes the closure of the union of the tetrahedra $T_{t}$, quantity (18) is equal to

$$
-\frac{1}{2} l\left(\dot{\beta}_{t}\right)+\frac{1}{2} l\left(p_{i}^{(t)} y_{i}^{(t)}\right) \frac{d}{d t} \theta_{T_{i}^{(t)}}\left(p_{i}^{(t)} y_{i}^{(t)}\right)+\frac{1}{2} l\left(q_{i}^{(t)} y_{i}^{(t)}\right) \frac{d}{d t} \theta_{T_{i}^{(t)}}\left(q_{i}^{(t)} y_{i}^{(t)}\right),
$$

where the negative sign comes from the fact that $F_{i}^{(t)}$ now occurs with the opposite orientation. Combining (22) and (28) yields that the infinite sums (13) and (19) add up to the finite sum

$$
\begin{aligned}
l\left(p_{i}^{(t)}\right. & \left.y_{i}^{(t)}\right) \frac{d}{d t} \theta_{H_{t}\left(R_{i}^{(t)} \times[0,1]\right)}\left(p_{i}^{(t)} y_{i}^{(t)}\right) \\
& +l\left(q_{i}^{(t)} y_{i}^{(t)}\right) \frac{d}{d t} \theta_{H_{t}\left(R_{i}^{(t)} \times[0,1]\right)}\left(q_{i}^{(t)} y_{i}^{(t)}\right) .
\end{aligned}
$$

Similarly, many terms cancel out as we take the sum of all terms (9), (10), (14), (15), (16) and (18) over the finitely many rectangles $R_{i}^{(t)}$.

For the terms (9), (10) and (16), this occurs in term by term cancellations. Indeed, with finitely many exceptions, the edge $p_{t} q_{t}$ of the rectangle $f_{t}\left(R_{t}\right)$ corresponding to the component $R_{t}$ of $R_{i}^{(t)}-\lambda_{t}$ coincides with an edge $p_{t}^{\prime} q_{t}^{\prime}$ or $r_{t}^{\prime} s_{t}^{\prime}$ of a rectangle $f_{t}\left(R_{t}^{\prime}\right)$ corresponding to a component $R_{t}^{\prime}$ of $R_{j}^{(t)}-\lambda_{t}$, for some rectangle $R_{j}^{(t)}$ possibly (and usually) different from $R_{i}^{(t)}$. The exceptions occur for those $p_{t} q_{t}$ which fall at the junction between two $f_{t}\left(R_{j}^{(t)}\right)$ and $f_{t}\left(R_{k}^{(t)}\right)$. If $p_{t} q_{t}$ coincides with such a $r_{t}^{\prime} s_{t}^{\prime}$, then

$$
\theta_{P_{t}}\left(p_{t} q_{t}\right)+\theta_{T_{t}}\left(p_{t} q_{t}\right)+\theta_{P_{t}^{\prime}}\left(r_{t}^{\prime} s_{t}^{\prime}\right)=2 \pi .
$$

It follows that the derivatives of these angles add up to 0 , and that the contributions of $p_{t} q_{t}=r_{t}^{\prime} s_{t}^{\prime}$ to (9-10) and (16) cancel out. A similar argument holds when $p_{t} q_{t}$ is equal to $p_{t}^{\prime} q_{t}^{\prime}$, and when $r_{t} s_{t}$ is equal to some $p_{t}^{\prime} q_{t}^{\prime}$ or $r_{t}^{\prime} s_{t}^{\prime}$. Thus as we take the sum of all terms (9), (10) and (16) over all rectangles $R_{i}^{(t)}$, we are left with only finitely many boundary terms. 
By an argument analogous to Lemma 7, the sum (14) can be interpreted in terms of the bending cocycle of the pleated fan that is the joint of $y_{i}^{(t)}$ and the arc $f_{t}([a, b] \times\{c\})$. A similar interpretation holds for the sum (18). It follows that, as we sum over all rectangles $R_{i}^{(t)}$, the terms (14) and (18) add up only to the sum of finitely many boundary terms.

Finally, it remains to consider the sum (15). The same arguments as in Lemma 7 express (15) as $\frac{1}{2}$ times the length of the derivative of the bending cocycle of the pleated rectangle $f_{t}\left(R_{i}^{(t)}\right)$, plus two boundary terms. Thus, as we sum over all rectangles $R_{i}^{(t)}$, the terms (15) add up to the sum of finitely many boundary terms and $\frac{1}{2} l\left(\dot{\beta}_{t}\right)$.

Combining these analyses, we conclude that

$$
d \operatorname{vol}\left(H_{t}\left(R^{(t)} \times[0,1]\right)\right) / d t
$$

is the sum of $\frac{1}{2} l\left(\dot{\beta}_{t}\right)$ and finitely many boundary terms corresponding to the edges of the polyhedral surface $H_{t}\left(\partial R^{(t)} \times[0,1]\right)$.

The volume bounded by $f_{t}$ is the sum of the volume of $H_{t}\left(R^{(t)} \times[0,1]\right)$, the volume of $H_{t}\left(\left(S-R^{(t)}\right) \times[0,1]\right)$, and the volume bounded by $g_{t}$. The last two of these volumes are bounded by polyhedral surfaces, and their variation is therefore given by Corollary 2, as the sum of finitely many boundary terms. We saw that the volume of $H_{t}\left(R^{(t)} \times[0,1]\right)$ is the sum of $\frac{1}{2} l\left(\dot{\beta}_{t}\right)$ and finitely many boundary terms. The boundary terms cancel out as in the proof of Corollary 2 , and we conclude that the derivative of the volume enclosed by $f_{t}$ is equal to $\frac{1}{2} l\left(\dot{\beta}_{t}\right)$.

This completes the proof of Theorem 3 when there are no cusps.

q.e.d.

Proof of Theorem 3 in the presence of cusps. Each cusp of $M_{0}$ has a neighborhood of the form $B / \Gamma_{1}$, where $B$ is a horoball of $\mathbb{H}^{3}$, and $\Gamma_{1}$ is a parabolic subgroup of $\Gamma$ that is isomorphic to $\mathbb{Z}$ (for a rank 1 cusp) or $\mathbb{Z}^{2}$ (for a rank 2 cusp). In addition, because the pleating locus of $f_{0}$ is compact, we can choose these cusp neighborhoods so that the intersection of $f_{0}$ with the cusp neighborhoods consists of finitely many totally geodesic annuli leading to the cusps. The fact that $f_{0}$ bounds a finite volume 3 -chain implies that, in each cusp neighborhood $B / \Gamma_{1}$, these annuli bound a locally finite 3 -chain relative to the boundary. If, in $f_{0}$, we chop off these annuli along piecewise geodesic simple closed curves, we suitably reconnect the pieces by polyhedral annuli, and we 
add a few polyhedral tori separating the rank-2 cusps from the rest of $M_{0}$, we obtain a compact surface $g_{0}$ which is (compactly) homologous to 0 in $M_{0}$, and whose pleating locus consists of $\lambda$ and finitely many polyhedral edges. The symmetric difference between $f_{0}$ and $g_{0}$ gives a polyhedral surface $h_{0}$ which bounds a finite volume locally finite $3-$ chain, and such that the 2-chain $f_{0}-g_{0}-h_{0}$ bounds a finite 3 -chain of volume 0 .

From $f_{t}$ in $M_{t}$, we can similarly define $g_{t}$ and $h_{t}$ in such a way that they depend differentiably on $t$. Then, the proof of Theorem 3 in the case without cusps immediately extends to show that the derivative at $t=0$ of the volume enclosed by $g_{t}$ is equal to

$$
\frac{1}{2} l_{0}\left(\dot{b}_{0}\right)+\frac{1}{2} \sum_{e} l_{0}(e) \dot{\theta}_{0}(e)
$$

where the sum is over the edges $e$ of the polyhedral part of $g_{0}$, and $\theta_{t}(e)$ is the external dihedral angle of $g_{t}$ at $e$. Note that every edge $e$ of $g_{t}$ occurs as an edge of $h_{t}$ with external dihedral angle $\pi-\theta_{t}(e)$. We can then invoke an easy extension of the Schläfli formula to polyhedral surfaces that have a compact set of edges and bound a finite volume locally finite 3-chain (Possible hint for a proof: cut off this locally finite extension by polyhedral surfaces that are arbitrarily close to the cusps, apply Theorem 2, and pass to the limit), which says that the derivative at $t=0$ of the volume enclosed by $h_{t}$ is equal to

$$
-\frac{1}{2} \sum_{e} l_{0}(e) \dot{\theta}_{0}(e)
$$

Since the 2 -chain $f_{t}-g_{t}-h_{t}$ bounds a volume 0 chain, adding up (30) and (31) completes the proof. q.e.d.

Remark 1. A more attractive approach to the proof of Theorem 3 would be to approximate the pleated surface $f_{t}$ by polyhedral surfaces $f_{t}^{\prime}$ and to show that, as the approximation gets better, the derivative given by the Schläfli formula for the volume enclosed by $f_{t}^{\prime}$ gets arbitrarily close to $\frac{1}{2} l_{t}\left(\dot{\beta}_{t}\right)$. This would decrease the cumbersome administration of building blocks in the above proof, and eliminate the consideration of internal edges whose contributions are eventually shown to cancel out. However, the author was unable to develop an approximation scheme where he could rigorously prove that this really happens.

Remark 2. Theorem 3 easily generalizes to the case where the pleating locus $\lambda$ of $f_{t}$ is non-compact. Indeed, there is a neighborhoood 
of the cusps of $S$ which meets only finitely many leaves of $\lambda$; see for instance [8, Theorem 4.2.8]. The bending cocycle

$$
b_{t} \in \mathcal{H}(\lambda ; \mathbb{R} / 2 \pi \mathbb{Z})
$$

has the additional property that, for every cusp, the $b_{t}$-masses of the finitely many leaf ends of $\lambda$ converging to that cusp add up to 0 . Since the same property holds for the derivative $\dot{b}_{0} \in \mathcal{H}(\lambda ; \mathbb{R})$, this enables one to define a finite length $l_{0}\left(\dot{b}_{0}\right)$ as the contributions of the leaf ends converging to the cusps cancel out in the limit. The proof of Theorem 3 immediately generalizes to show that the equality $\dot{V}_{0}=\frac{1}{2} l_{0}\left(\dot{b}_{0}\right)$ also holds in this case.

\section{Proof of the main theorem}

We now prove the main theorem.

Theorem 8. Let $M_{t}, t \in[0, \varepsilon[$, be a cusp-preserving deformation of the geometrically finite hyperbolic 3-manifold $M_{0}$. Let $b_{t} \in \mathcal{M L}\left(\partial C_{M_{0}}\right)$ be the bending measured geodesic lamination of the boundary $\partial C_{M_{t}}$ of the convex core of $M_{t}$ (using the convention that $\partial C_{M_{t}}$ is the unit normal bundle of $C_{M_{t}}$ when the convex core $C_{M_{t}}$ is 2-dimensional). Then, the volume $V_{t}$ of the convex core $C_{M_{t}}$ admits a right derivative at $t=0$, and

$$
\dot{V}_{0}=\frac{1}{2} l_{0}\left(\dot{b}_{0}\right),
$$

where $l_{0}\left(\dot{b}_{0}\right)$ is the length of the vector $\dot{b}_{0}$ tangent to the family of bending measured laminations $b_{t}$ (whose existence is proved by [6]).

Proof of Theorem 8 when there are no cusps. Let $S$ denote the surface $\partial C_{M_{0}}$. Then, the bending measured geodesic laminations $b_{t}$ belong to the space $\mathcal{M L}(S)$ of measured geodesic laminations on $S$. Let $f_{t}: S \rightarrow M_{t}$ be the pleated surface whose image is the boundary $\partial C_{M_{t}}$, and let $\lambda_{t}$ be a pleating locus for $f_{t}$ which is maximal among compact geodesic laminations. Note that $\lambda_{t}$ contains the support of the bending measured geodesic lamination $b_{t}$.

We first prove Theorem 8 under the additional assumption that, as $t$ tends to $0^{+}$, the geodesic lamination $\lambda_{t}$ tends to a geodesic lamination $\lambda$ for the Hausdorff topology. In particular, this is always the case when the support of $b_{0}$ is maximal. The fact that the $\lambda_{t}$ are maximal imply that $\lambda$ is also maximal. 
As in [4], we identify the tangent vector $\dot{b}_{0}$ with a compact geodesic lamination endowed with a certain transverse cocycle. Note that the support of $\dot{b}_{0}$ is necessarily contained in the Hausdorff limit $\lambda$ (see for instance $[4, \S 2]$ ), so that $\dot{b}_{0}$ can be interpreted as a transverse cocycle for $\lambda$. For every $t$, let $f_{t}^{\prime}: S \rightarrow M_{t}$ be the (unique) pleated surface with pleating locus $\lambda$. We will prove Theorem 8 by comparing the volume $V_{t}$ of $C_{M_{t}}$ to the volume $V_{t}^{\prime}$ enclosed by $f_{t}^{\prime}$ in $M_{t}$. Note that $f_{0}^{\prime}=f_{0}$, but that the pleating locus $\lambda_{t}$ of $f_{t}$ varies while the pleating locus $\lambda$ of $f_{t}^{\prime}$ is constant.

We will use the Stokes Formula to compare the volumes respectively enclosed by the pleated surfaces $f_{t}$ and $f_{t}^{\prime}$. Since the theorem is otherwise trivial by Mostow's Rigidity Theorem, we can assume that the $M_{t}$ are non-compact. Then, $H^{3}\left(M_{t} ; \mathbb{R}\right)=0$, and there exists a differential 2 -form $\omega_{t}$ such that $d \omega_{t}$ is the volume form of $M_{t}$. We first show that the $\omega_{t}$ can be chosen to depend differentiably on $t$.

Lemma 9. There is a family of differential 2 -forms $\omega_{t}$ such that $d \omega_{t}$ coincides with the volume form of $M_{t}$ on a neighborhood of the convex core $C_{M_{t}}$, and such that $\omega_{t}$ depends differentiably on $t$ in the following sense: If we pull back the form $\omega_{t}$ on $M_{t} \cong \mathbb{H}^{3} / \rho_{t}(\Gamma)$ to a form $\widetilde{\omega}_{t}$ on $\mathbb{H}^{3}$, then $\widetilde{\omega}_{t}$ depends differentiably on $t$.

Proof. We will use a celebrated result of J. Moser [14]. By [13, $\S 9]$, there is for every $t$ a diffeomorphism $\varphi_{t}: M_{0} \rightarrow M_{t}$. In addition, the proof of this result makes it clear that $\varphi_{t}$ can be chosen to depend differentiably on $t$, in the sense that it lifts to a family of diffeomorphisms $\widetilde{\varphi}_{t}: \mathbb{H}^{3} \rightarrow \mathbb{H}^{3}$ which depend differentiably on $t$.

If $\nu_{t}$ denotes the volume form of $M_{t}$, the $\varphi_{t}^{*}\left(\nu_{t}\right)$ give a 1-parameter family of volume forms on $M_{0}$ which are all cohomologous (to 0 ). Then, Moser's Lemma [14] asserts that these volume forms are all isotopic: For every compact $K \subset M_{0}$, there are diffeomorphisms $\psi_{t}: M_{0} \rightarrow M_{0}$ depending differentiably on $t$ such that $\varphi_{t}^{*}\left(\nu_{t}\right)=\psi_{t}^{*}\left(\nu_{0}\right)$ on $K$ and $\psi_{0}=$ Id (Moser's proof provides a vector field, and one needs to restrict to a compact subset $K$ to integrate it).

Pick a 2 -form $\omega_{0}$ on $M_{0}$ such that $d \omega_{0}=\nu_{0}$, and a compact $K \subset$ $M_{0}$ which contains a neighborhood of all the $\varphi_{t}^{-1}\left(C_{M_{t}}\right)$. Then $\omega_{t}=$ $\left(\varphi_{t}^{-1}\right)^{*} \psi_{t}^{*}\left(\omega_{0}\right)$ satisfies the properties required. q.e.d.

For every $t$, the image of $f_{t}^{\prime}$ is contained in $C_{M_{t}}$ and, because the complement $M-\partial C_{M_{t}}$ is homeomorphic to a product $S \times \mathbb{R}$ (see for instance $[10, \S 1]), f_{t}^{\prime}$ is homologous to 0 in $C_{M_{t}}$. Stokes' Formula then 
shows that the volumes respectively enclosed by $f_{t}$ and $f_{t}^{\prime}$ are equal to

$$
V_{t}=\int_{S} f_{t}^{*}\left(\omega_{t}\right) \text { and } V_{t}^{\prime}=\int_{S}\left(f_{t}^{\prime}\right)^{*}\left(\omega_{t}\right)
$$

(We let the reader check, for instance through an approximation by polyhedral surfaces, that Stokes' formula holds for pleated surfaces).

By a suitable partition of unity, $\omega_{t}$ coincides on a neighborhood of $C_{M_{t}}$ with a finite sum $\sum_{i=1}^{n} \omega_{t}^{(i)}$, where each $\omega_{t}^{(i)}$ is the push forward of a compactly supported 2-form $\widetilde{\omega}_{t}^{(i)}$ on $\mathbb{H}^{3}$. In addition, using the diffeomorphisms $\varphi_{t}: M_{0} \rightarrow M_{t}$, we can arrange that the $\widetilde{\omega}_{t}^{(i)}$ depend differentiably on $t$. Consider the covering $\widehat{S} \rightarrow S$, pull back of the covering $\mathbb{H}^{3} \rightarrow M_{0}$ by the map $f_{0}: S \rightarrow M_{0}$, and the canonical lift $\widehat{f_{0}}: \widehat{S} \rightarrow \mathbb{H}^{3}$. Recall that $\widehat{S}$ consists of all pairs $(x, y) \in S \times \mathbb{H}^{3}$ such that $f_{0}(x)$ is equal to the projection of $y$ in $M_{0}$. In particular, the homotopy from $f_{0}$ to $f_{t}$ and $f_{t}^{\prime}$ uniquely defines lifts $\widehat{f}_{t}, \widehat{f}_{t}^{\prime}: \widehat{S} \rightarrow \mathbb{H}^{3}$. Note that, because the group $\rho_{t}\left(\pi_{1}(S)\right)$ acts properly discontinuously on $\mathbb{H}^{3}$, the pleated surfaces $\widehat{f}_{t}$ and $\widehat{f}_{t}^{\prime}$ are proper. The definitions are specially designed so that

$$
V_{t}=\sum_{i=1}^{n} \int_{\widehat{S}} \widehat{f}_{t}^{*}\left(\widetilde{\omega}_{t}^{(i)}\right) \text { and } V_{t}^{\prime}=\sum_{i=1}^{n} \int_{\widehat{S}}\left(\widehat{f}_{t}^{\prime}\right)^{*}\left(\widetilde{\omega}_{t}^{(i)}\right)
$$

Lemma 10. For every compactly supported differential 2-form $\widetilde{\omega}$ on $\mathbb{H}^{3}$, the following two right derivatives exist and are equal:

$$
\frac{d}{d t^{+}} \int_{\widehat{S}} \widehat{f}_{t}^{*}(\widetilde{\omega})_{\mid t=0}=\frac{d}{d t^{+}} \int_{\widehat{S}}\left(\widehat{f}_{t}^{\prime}\right)^{*}(\widetilde{\omega})_{\mid t=0} .
$$

Proof. It clearly suffices to restrict attention to each component $\widehat{S}_{1}$ of $\widehat{S}$, projecting to a component $S_{1}$ of $S$. Because $f_{t}$ and $f_{t}^{\prime}$ depend continuously on $t$, we can use a partition of unity to assume, without loss of generality, that there is a compact subset $\widetilde{B}$ of the universal covering $\widetilde{S}$ of $\widehat{S}_{1}$ (and $S_{1}$ ) such that the projection $\widetilde{S} \rightarrow \widehat{S}_{1}$ is injective on $\widetilde{B}$, and such that the intersection of the support of $\widetilde{\omega}$ with each $\widehat{f}_{t}\left(\widehat{S}_{1}\right)$ or $\widehat{f}_{t}^{\prime}\left(\widehat{S}_{1}\right)$ is contained in $\widetilde{f}_{t}(\widetilde{B})$ or $\widetilde{f}_{t}^{\prime}(\widetilde{B})$, respectively, where $\widetilde{f}_{t}$ and $\widetilde{f}_{t}^{\prime}$ denote the composition of the projection $\widetilde{S} \rightarrow \widehat{S}_{1}$ with the restrictions of $\widehat{f}_{t}$ and $\widehat{f}_{t}^{\prime}$ to $\widehat{S}_{1}$. We now have

$$
\int_{\widehat{S}_{1}} \widehat{f}_{t}^{*}(\widetilde{\omega})=\int_{\widetilde{B}} \widetilde{f}_{t}^{*}(\widetilde{\omega}) \text { and } \int_{\widehat{S}_{1}}\left(\widehat{f}_{t}^{\prime}\right)^{*}(\widetilde{\omega})=\int_{\widetilde{B}}\left(\widetilde{f_{t}^{\prime}}\right)^{*}(\widetilde{\omega}) \text {. }
$$


Then, the property of Lemma 10 is essentially proved in $[6, \S 2]$, where we compare the two pleated surfaces $\widetilde{f}_{t}, \widetilde{f}_{t}^{\prime}: \widetilde{S} \rightarrow \mathbb{H}^{3}$. However, minor adjustments are necessary because the pull back metrics $m_{t}$ and $m_{t}^{\prime}$ induced on $S_{1}$ by $f_{t}$ and $f_{t}^{\prime}$ may be different, and also because we used a normalization which we now have to take into account.

Pick a base frame at some point of $\widetilde{S}-\widetilde{\lambda}$, or more precisely at some point of the complement in $\widetilde{S}$ of the $m_{0}$-geodesic lamination corresponding to $\lambda \cap S_{1}$. Changing the identification of the universal covering of $M_{t}$ with $\mathbb{H}^{3}$ (and conjugating the representation $\rho_{t}: \Gamma \rightarrow \operatorname{Isom}^{+}\left(\mathbb{H}^{3}\right)$ accordingly), we can arrange that each $\tilde{f}_{t}$ coincides with $\tilde{f}_{0}$ at this base frame.

For every $t$, there is a unique isometry $A_{t} \in \mathrm{Isom}^{+}\left(\mathbb{H}^{3}\right)$ such that the pleated surface $\widetilde{g}_{t}^{\prime}=A_{t} \circ \widetilde{f}_{t}^{\prime}$ also coincides with $\widetilde{f}_{0}$ at the base frame. Note that $\hat{g}_{t}^{f}$ has the same pull back metric $m_{t}^{\prime}$ and the same bending transverse cocycle $b_{t}^{\prime} \in \mathcal{H}\left(\lambda \cap S_{1} ; \mathbb{R} / 2 \pi \mathbb{Z}\right)$ as $\widetilde{f_{t}^{\prime}}$.

As an interpolation between $\widetilde{f}_{t}$ and $\widetilde{g}_{t}^{\prime}$, consider the pleated surface $\tilde{g}_{t}^{\prime \prime}: \widetilde{S} \rightarrow \mathbb{H}^{3}$ which has the same pull back metric $m_{t}$ as $\widetilde{f}_{t}$, but has the same pleating locus $\lambda$ and the same bending transverse cocycle $b_{t}^{\prime} \in$ $\mathcal{H}\left(\lambda \cap S_{1} ; \mathbb{R} / 2 \pi \mathbb{Z}\right)$ as $\widetilde{g}_{t}^{\prime}$, and which coincides with $\widetilde{f}_{0}$ at the base frame.

Since $\widetilde{f}_{t}$ and $\widetilde{f}_{t}^{\prime}$ are equivariant with respect to the same representation $\rho_{t}: \Gamma \rightarrow$ Isom $^{+}\left(\mathbb{H}^{3}\right)$, from Propositions 5 and 10 of [6] it follows that $\dot{m}_{0}=\dot{m}_{0}^{\prime}$ and $\dot{b}_{0}=\dot{b}_{0}^{\prime} \in \mathcal{H}\left(\lambda \cap S_{1} ; \mathbb{R}\right)$. In particular, the two pleated surfaces $\widetilde{f}_{t}$ and $\widetilde{g}_{t}^{\prime \prime}$ coincide when $t=0$, coincide for every $t$ at the base frame, have the same pull back metric $m_{t}$, and have the same variation $\dot{b}_{0}=\dot{b}_{0}^{\prime}$ of bending data at $t=0$. Under these conditions, we show in $[6, \S 2]$ that $\widetilde{f}_{t}$ and $\widetilde{g}_{t}^{\prime \prime}$ are infinitesimally close as $t$ tends to 0 , and this uniformly on compact subsets of $\widetilde{S}-\widetilde{\lambda}$. In particular, the arguments of $[6, \S 2]$, and most notably the key growth estimate of $[6$, Lemma 7], show that

$$
\frac{d}{d t^{+}} \int_{\widetilde{B}} \widetilde{f}_{t}^{*}(\widetilde{\omega})_{\mid t=0}=\frac{d}{d t^{+}} \int_{\widetilde{B}}\left(\widetilde{g}_{t}^{\prime \prime}\right)^{*}(\widetilde{\omega})_{\mid t=0}
$$

Similarly, the pleated surfaces $\widetilde{g}_{t}^{\prime}$ and $\widetilde{g}_{t}^{\prime \prime}$ coincide when $t=0$, coincide for every $t$ at the base frame, have the same pleating locus $\lambda \cap S_{1}$ and the same bending transverse cocycle $b_{t}^{\prime} \in \mathcal{H}\left(\lambda \cap S_{1} ; \mathbb{R} / 2 \pi \mathbb{Z}\right)$, and have the same variation $\dot{m}_{0}=\dot{m}_{0}^{\prime}$ for their pull back metrics. The much easier arguments of $[5, \S 5]$ show that these two surfaces are infinitesi- 
mally close, and that

$$
\frac{d}{d t} \int_{\widetilde{B}}\left(\widetilde{g}_{t}^{\prime}\right)^{*}(\widetilde{\omega})_{\mid t=0}=\frac{d}{d t} \int_{\widetilde{B}}\left(\widetilde{g}_{t}^{\prime \prime}\right)^{*}(\widetilde{\omega})_{\mid t=0}
$$

By construction,

$$
\int_{\widetilde{B}}\left(\widetilde{f}_{t}^{\prime}\right)^{*}(\widetilde{\omega})=\int_{\widetilde{B}}\left(\widetilde{g}_{t}^{\prime}\right)^{*}\left(A_{t}^{-1}\right)^{*}(\widetilde{\omega})
$$

Note that $\widetilde{g}_{t}^{\prime}: \widetilde{S} \rightarrow \mathbb{H}^{3}$ is equivariant with respect to the representation $\rho_{t}^{\prime}=A_{t}^{-1} \rho_{t} A_{t}: \Gamma \rightarrow \operatorname{Isom}^{+}\left(\mathbb{H}^{3}\right)$, while $\widetilde{f}_{t}$ is equivarant with respect to $\rho_{t}$. From the fact that these two pleated surfaces are infinitesimally close as $t$ tends to $0^{+}$, we conclude that $\dot{\rho}_{0}=\dot{\rho}_{0}^{\prime}$. Looking at fixed points, for instance, it follows that $\dot{A}_{0}=0$, while $A_{0}=$ Id by construction. Combining (36-38) gives

$$
\begin{aligned}
\frac{d}{d t^{+}} \int_{\widetilde{B}}\left(\widetilde{f}_{t}^{\prime}\right)^{*}(\widetilde{\omega})_{\mid t=0} & =\frac{d}{d t^{+}} \int_{\widetilde{B}}\left(\widetilde{g}_{t}^{\prime}\right)^{*}(\widetilde{\omega})_{\mid t=0} \\
& =\frac{d}{d t^{+}} \int_{\widetilde{B}} \widetilde{f}_{t}^{*}(\widetilde{\omega})_{\mid t=0}
\end{aligned}
$$

which concludes the proof by (35). q.e.d.

From (33) and the existence of the derivative in Lemma 10, we find that

$$
\dot{V}_{0}=\sum_{i=1}^{n} \frac{d}{d t^{+}} \int_{\widehat{S}} \widehat{f}_{t}^{*}\left(\widetilde{\omega}_{0}^{(i)}\right)_{\mid t=0}+\sum_{i=1}^{n} \int_{\widehat{S}} \widehat{f}_{0}^{*}\left(\frac{d}{d t^{+}} \widetilde{\omega}_{t}^{(i)} \mid t=0\right),
$$

where the chain rule is justified by the fact that the map $t \mapsto \int_{\widehat{S}} \widehat{f_{t}^{*}}(\widetilde{\omega})$ is continuous, uniformly on compact sets for the 2 -form $\widetilde{\omega}$. The last term of $(40)$ is equal to

$$
\int_{S} f_{0}^{*}\left(\sum_{i=1}^{n} \frac{d}{d t^{+}} \omega_{t}^{(i)} \mid t=0\right)=\int_{S} f_{0}^{*}\left(\dot{\omega}_{0}\right)=\int_{C_{M_{0}}} d \dot{\omega}_{0}=0,
$$

since the lift of $d \omega_{t}$ to $\mathbb{H}^{3}$ is constant, equal to the volume form of $\mathbb{H}^{3}$. Therefore,

$$
\dot{V}_{0}=\sum_{i=1}^{n} \frac{d}{d t^{+}} \int_{\widehat{S}} \widehat{f}_{t}^{*}\left(\widetilde{\omega}_{0}^{(i)}\right)_{\mid t=0} .
$$


Similarly,

$$
\dot{V}_{0}^{\prime}=\sum_{i=1}^{n} \frac{d}{d t^{+}} \int_{\widehat{S}}\left(\widehat{f}_{t}^{\prime}\right)^{*}\left(\widetilde{\omega}_{0}^{(i)}\right)_{\mid t=0} .
$$

Combining (42), (43), Lemma 10 and Theorem 3 yields

$$
\dot{V}_{0}=\dot{V}_{0}^{\prime}=\frac{1}{2} l_{0}\left(\dot{b}_{0}^{\prime}\right)=\frac{1}{2} l_{0}\left(\dot{b}_{0}\right)
$$

which completes the proof of Theorem 8 under the assumption that, as $t$ tends to $0^{+}$, the geodesic lamination $\lambda_{t}$ converge to a geodesic lamination $\lambda$ for the Hausdorff topology.

In the general case, choose a sequence $t_{n}$ converging to 0 such that $\lambda_{t_{n}}$ converges to some geodesic lamination $\lambda$. Then, the arguments of the special case apply to show that $\left(V_{t_{n}}-V_{0}\right) / t_{n}$ tends to $\frac{1}{2} l_{0}\left(\dot{b}_{0}\right)$ as $n$ tends to infinity. Since this holds for any such sequence $t_{n}$, we conclude that $\dot{V}_{0}=\frac{1}{2} l_{0}\left(\dot{b}_{0}\right)$ in the general case as well. q.e.d.

Proof of Theorem 8 in the presence of cusps. When the manifolds $M_{t}$ have cusps, the boundary $\partial C_{M_{t}}$ is totally geodesic near the cusps of $M_{t}$. Therefore, we can use the same technique as in the proof of Theorem 3, and chop off pieces of $C_{M_{t}}$ by polyhedral surfaces near the cusps. The proof in this case then follows from the proof in the case without cusps, as for Theorem 3 . q.e.d.

\section{Convex cores with totally geodesic boundary}

We conclude this paper with an application of Theorem 8.

Corollary 11. Let $M$ be a geometrically finite hyperbolic 3-manifold whose convex core $C_{M}$ has totally geodesic boundary, but is not 2dimensional. Consider the volumes of the convex cores of the cusppreserving deformations of $M$. Then $M$ is a strict local minimum for this volume function.

Proof. Let $\mathcal{Q D}(M)$ denote the space of hyperbolic 3-manifolds obtained by cusp-preserving deformations of $M$. Theorem 8 determines the tangent map $T_{M} V: T_{M} \mathcal{Q D}(M) \rightarrow \mathbb{R}$ of the function $V:$ $\mathcal{Q D}(M) \rightarrow \mathbb{R}^{+}$defined by consideration of the volumes of convex cores, in terms of the tangent map of the bending measured lamination map $\beta$ : $\mathcal{Q D}(M) \rightarrow \mathcal{M L}\left(\partial C_{M}\right)$ analyzed in [6]. Namely, $T_{M} V=\frac{1}{2} l_{\partial C_{M}} \circ T_{M} \beta$, 
where $l_{\partial C_{M}}$ denotes the length function on the hyperbolic surface $\partial C_{M}$. Note that these tangent maps are not necessarily linear; see $[6, \S 1]$.

Suppose that $M$ is not a strict local minimum for the volume function $V$. Then, from a sequence $M_{n} \in \mathcal{Q D}(M)$ converging to $M$ with $V\left(M_{n}\right) \leqslant V(M)$, we can construct a non-zero tangent vector $v \in T_{M} \mathcal{Q D}(M)$ such that $T_{M} V(v) \leqslant 0$. Now, Theorem 8 says that $T_{M} V(v)=\frac{1}{2} l_{\partial C_{M}}\left(T_{M} \beta(v)\right)$. Since the boundary of $C_{M}$ is totally geodesic, $\beta(M)=0$ and $T_{M} \beta(v)$ is a vector tangent to $\mathcal{M L}(S)$ at 0 . By [4, Theorem 21], $T_{M} \beta(v)$ is therefore a geodesic lamination with a transverse (positive) measure. The main consequence of this is that $T_{M} \beta(v)$ has positive length if $T_{M} \beta(v) \neq 0$. Since $T_{M} V(v) \leqslant 0$, we must have $T_{M} \beta(v)=0$. The proof is thus completed by the following lemma.

Lemma 12. Under the hypotheses of Corollary 11, there is no nonzero tangent vector $v \in T_{M} \mathcal{Q D}(M)$ such that $T_{M} \beta(v)=0$. In other words, there is no infinitesimal deformation of $M$ which infinitesimally keeps the boundary of $C_{M}$ flat.

Proof. Suppose there is such a tangent vector $v$. Consider the manifold $D M$ obtained by taking the double of $C_{M}$ along its boundary. Namely, $D M$ is obtained by gluing two copies of $C_{M}$ along their boundaries by the natural identification. Because $\partial C_{M}$ is totally geodesic, the hyperbolic metric of $M$ gives a finite volume complete hyperbolic metric on $D M$.

First suppose that, in addition, there is a 1-parameter family of deformations $M_{t}, t \in\left[0, \varepsilon\left[\right.\right.$, such that $M_{0}=M, \dot{M}_{0}=v$ and the convex cores $C_{M_{t}}$ all have totally geodesic boundary. Then, the hyperbolic manifolds $D M_{t}$ give a non-trivial cusp-preserving deformation of $D M$, which is excluded by Mostow's Rigidity Theorem [15]. The general case follows this heuristic line of argument.

In general, the fact that $T_{M} \beta(v)=0$ only means that, for a 1parameter family of deformations $M_{t}, t \in\left[0, \varepsilon\left[\right.\right.$, with $M_{0}=M$ and $\dot{M}_{0}=v$, the bending measured geodesic lamination $b_{t} \in \mathcal{M L}\left(\partial C_{M}\right)$ of $M_{t}$ is such that $b_{0}=0$ and $\dot{b}_{0}=0$. We will use an infinitesimal version of the heuristic argument, based on the Calabi-Weil Rigidity Theorem [7], [22] which is an infinitesimal version of Mostow rigidity. Indeed, interpreting $\mathcal{Q D}(M)$ as a space of representations

$$
\rho: \pi_{1}(M) \rightarrow \operatorname{Isom}^{+}\left(\mathbb{H}^{3}\right),
$$

the Weil machinery (see for instance [17]) expresses the tangent space of $\mathcal{Q D}(M)$ at $M$ as a subspace of the cohomology group $H^{1}\left(\pi_{1}(M), \mathrm{Ad}\right)$, 
where Ad denotes the adjoint representation of $\pi_{1}(M)$ in the Lie algebra of Isom ${ }^{+}\left(\mathbb{H}^{3}\right)$ defined by the holonomy of $M$. The reason why $T_{M} \mathcal{Q D}(M)$ is only a subspace of $H^{1}\left(\pi_{1}(M), \mathrm{Ad}\right)$ is that we restrict attention to cusp-preserving deformations. If $S$ is a component of $\partial C_{M}$, let $M_{S}$ be the covering of $M$ with $\pi_{1}\left(M_{S}\right)=\pi_{1}(S)$. The metric of $M$ lifts to a Fuchsian hyperbolic metric on $M_{S}$. Because $T_{M} \beta(v)=0$, [6, Proposition 5] shows that the differential of the restriction map $\mathcal{Q D}(M) \rightarrow \mathcal{Q D}\left(M_{S}\right)$ sends $v$ to a vector

$$
v_{S} \in T_{M_{S}} \mathcal{Q D}\left(M_{S}\right)=H^{1}\left(\pi_{1}(S), \mathrm{Ad}\right)
$$

that is tangent to the submanifold of Fuchsian deformations of $M_{S}$. In particular, $v_{S}$ is invariant under the automorphism of $T_{M_{S}} \mathcal{Q D}\left(M_{S}\right)$ induced by the isometry that reflects $M_{S}$ across the totally geodesic surface $C_{M_{S}}$. Since this holds for every component $S$ of $\partial C_{M}$, a MayerVietoris type argument shows that $v$ provides a non-trivial element of $H^{1}\left(\pi_{1}(D M), \mathrm{Ad}\right)$ which keeps the cusps parabolic. However, the Calabi-Weil Rigidity Theorem [7], [22], as improved by Garland [11] for the case with cusps, says that there is no such non-trivial element of $H^{1}\left(\pi_{1}(D M), \mathrm{Ad}\right)$. q.e.d.

This concludes the proof of Corollary 11 . q.e.d.

When $C_{M}$ is 2-dimensional, $M$ is of course a global minimum for $V$ since $V(M)=0$. In this case, $M$ is Fuchsian or twisted Fuchsian, and there are many deformations which keep the convex core 2-dimensional. Therefore, $M$ is only a weak local minimum.

There presumably is a converse to Corollary 11: If $M$ is a local minimum for the convex core volume function $V$, then the boundary $\partial C_{M}$ is totally geodesic. This would follow from Theorem 8 and a conjectural extension to convex cores of Cauchy's Rigidity Theorem for polyhedra; compare [18].

\section{References}

[1] D. V. Alekseevskij, E. B. Vinberg \& A. S. Solodovnikov, Geometry of spaces of constant curvature, Geometry II (ed. E.B. Vinberg), Encyclopaedia Math. Sci., Vol. 29, Springer, Berlin, 1993, 1-138.

[2] J. S. Birman \& C. Series, Geodesics with bounded intersection numbers on surfaces are sparsely distributed, Topology 24 (1985) 217-225. 
[3] F. Bonahon, Transverse Hölder distributions for geodesic laminations, Topology 36 (1997) 103-122.

[4] - Geodesic laminations with transverse Hölder distributions, Ann. Sci. École Norm. Sup. 30 (1997) 205-240.

[5] _ Shearing hyperbolic surfaces, bending pleated surfaces, and the Thurston symplectic form, Ann. Fac. Sci. Toulouse Math. 5 (1996 ) 233-297.

[6] - Variations of the boundary geometry of 3-dimensional hyperbolic convex cores, J Differential Geom. 50 (1998) 1-24.

[7] E. Calabi, On compact riemannian manifolds with constant curvature. I, Proc. Sympos. Pure Math., Vol. III, Amer. Math. Soc., Providence, 1961, 155-180.

[8] R. D. Canary, D. B. A. Epstein \& P. Green, Notes on notes of Thurston, Analytical and Geometrical aspects of Hyperbolic space (ed. D.B.A. Epstein), L. M. S. Lecture Notes Series, Vol. 111, Cambridge Univ. Press, Cambridge, 1987, 3-92.

[9] A. Casson \& S.A. Bleiler, Automorphisms of surfaces after Thurston and Nielsen, Cambridge Univ. Press, Cambridge, 1988.

[10] D. B. A. Epstein \& A. Marden, Convex hulls in hyperbolic spaces, a theorem of Sullivan, and measured pleated surfaces, Analytical and geometric aspects of hyperbolic space (ed. D.B.A. Epstein), London Math. Soc. Lecture Note Ser., Vol. 111, Cambridge Univ. Press, Cambridge, 1986, 113-253.

[11] H. Garland, A rigidity theorem for discrete subgroups, Trans Amer. Math. Soc. 129 (1967) 1-25.

[12] H. Kneser, Der Simplexinhalt in der nichteuklidischen Geometrie, Deutsche Math. 1 (1936) 337-340.

[13] A. Marden, The geometry of finitely generated Kleinian groups, Ann. of Math. 99 (1974) 383-462.

[14] J. Moser, On the volume elements on a manifold, Trans. Amer. Math. Soc. 120 (1965) 286-294.

[15] G. D. Mostow, Strong rigidity of locally symmetric spaces, Ann. of Math. Stud., Vol. 78, Princeton Univ. Press, Princeton, 1973.

[16] R. C. Penner \& J. L. Harer, Combinatorics of train tracks, Ann. of Math. Stud. Vol. 125, Princeton Univ. Press, Princeton, 1992.

[17] M. S. Ragunathan, Discrete subgroups of Lie groups, Ergeb. Math. Grenzgeb. Vol. 58, Springer, Berlin, 1972.

[18] I. Rivin \& C. D. Hodgson, A characterization of convex polyhedra in hyperbolic 3-space, Invent. Math. 111 (1993) 77-111; Corrigendum, Invent. Math. 117 (1994) 359. 
[19] L. Schläfli, On the multiple integral $\iint \cdots \int d x d y \ldots d z$ whose limits are $p_{1}=a_{1} x+$ $b_{1} y+\cdots+h_{1} z>0, p_{2}>0, \ldots, p_{n}>0$ and $x^{2}+y^{2}+\cdots+z^{2}=1$, Q. J. Math 2 (1858) 269-300; idem, Q. J. Math 3 (1860) 54-68; idem, Q. J. Math. 3 (1860) 97-108; see also [20, pp.219-270].

[20] — Gesammelte Mathematische Abhandlungen, Band II, Birkhäuser, Basel, 1950.

[21] W. P. Thurston, The topology and geometry of 3-manifolds, Princeton Univ., Lecture Notes, 1976-79.

[22] A. Weil, On discrete subgroups of Lie groups. II, Ann. of Math. 75 (1962) 578-602.

University of SOUthern CaLifornia 